\title{
The Spin Evolution of Fast-rotating, Magnetized Super-Chandrasekhar White Dwarfs in the Aftermath of White Dwarf Mergers
}

\author{
L. Becerra ${ }^{1,2}$, J. A. Rueda ${ }^{1,2,3}$ (D) P. Lorén-Aguilar ${ }^{4}$ (D) , and E. García-Berro ${ }^{5,6}$ (1) \\ ${ }^{1}$ Dipartimento di Fisica, Sapienza Università di Roma, P.le Aldo Moro 5, I-00185 Rome, Italy \\ 2 ICRANet, P.zza della Repubblica 10, I-65122 Pescara, Italy \\ ${ }^{3}$ ICRANet-Rio, Centro Brasileiro de Pesquisas Físicas, Rua Dr. Xavier Sigaud 150, 22290-180 Rio de Janeiro, Brazil \\ ${ }^{4}$ School of Physics, University of Exeter, Stocker Road, Exeter EX4 4QL, UK \\ ${ }^{5}$ Departament de Física, Universitat Politècnica de Catalunya, c/Esteve Terrades, 5, E-08860 Castelldefels, Spain \\ ${ }^{6}$ Institut d'Estudis Espacials de Catalunya, Ed. Nexus-201, c/Gran Capità 2-4, E-08034 Barcelona, Spain \\ Received 2016 September 1; revised 2018 March 30; accepted 2018 April 3; published 2018 April 25
}

\begin{abstract}
The evolution of the remnant of the merger of two white dwarfs is still an open problem. Furthermore, few studies have addressed the case in which the remnant is a magnetic white dwarf with a mass larger than the Chandrasekhar limiting mass. Angular momentum losses might bring the remnant of the merger to the physical conditions suitable for developing a thermonuclear explosion. Alternatively, the remnant may be prone to gravitational or rotational instabilities, depending on the initial conditions reached after the coalescence. Dipole magnetic braking is one of the mechanisms that can drive such losses of angular momentum. However, the timescale on which these losses occur depends on several parameters, like the strength of the magnetic field. In addition, the coalescence leaves a surrounding Keplerian disk that can be accreted by the newly formed white dwarf. Here we compute the postmerger evolution of a super-Chandrasekhar magnetized white dwarf taking into account all the relevant physical processes. These include magnetic torques acting on the star, accretion from the Keplerian disk, the threading of the magnetic field lines through the disk, and the thermal evolution of the white dwarf core. We find that the central remnant can reach the conditions suitable to develop a thermonuclear explosion before other instabilities (such as the inverse beta-decay instability or the secular axisymmetric instability) are reached, which would instead lead to gravitational collapse of the magnetized remnant.
\end{abstract}

Key words: accretion, accretion disks - stars: magnetic field - stars: rotation - supernovae: general - white dwarfs

\section{Introduction}

Type Ia supernovae (SNe Ia) are one of the most energetic explosive events in the cosmos. Since there is a wellestablished relationship between their intrinsic brightnesses and the shapes of their light curves (Phillips 1993) and, moreover, because they are so luminous that they can be detected at very large distances, $\mathrm{SNe}$ Ia can be used as standardizable cosmological candles. This has opened a new era in cosmology and has enabled us to measure the acceleration of the universe (Riess et al. 1998; Perlmutter et al. 1999). Additionally, SNe Ia play an important role in modern cosmology, since they allow us to probe the fundamental physical theories underlying dark energy-see, for instance, Weinberg et al. (2013) for a recent review on this interesting topic.

Nevertheless, despite the importance of SNe Ia, we still do not know the nature of their progenitor systems. Actually, this has remained a long-standing mystery for several decades. We do know that the outburst is driven by the explosion of a carbon-oxygen white dwarf in a binary system, but we do not know the precise mechanism that destabilizes the white dwarf. Several hypotheses have been put forward over the years, and most likely SNe Ia may have a diversity of progenitors. In the following we superficially describe the possible evolutionary channels leading to an SN Ia. In the so-called single-degenerate (SD) channel a white dwarf in a binary system accretes matter from a nondegenerate companion and explodes as it approaches the Chandrasekhar limiting mass-see, for instance, Han \& Podsiadlowski (2004) for a recent discussion. In the double-degenerate (DD) scenario two white dwarf members of a close binary system lose angular momentum and energy through the radiation of gravitational waves, and a merger occurs (Iben \& Tutukov 1984; Webbink 1984). Another possible scenario is the core-degenerate (CD) scenario. Within this formation channel a hot core is formed at the end of the common envelope phase of the binary system (Livio \& Riess 2003; Kashi \& Soker 2011), and the merger of the core of the asymptotic giant branch (AGB) star and a secondary white dwarf powers the explosion-see Aznar-Siguán et al. (2015) for a scrocess. Another recently proposed pathway is the white dwarf-white dwarf collisional scenario, in which two white dwarfs collide-see Aznar-Siguán et al. (2013) for a recent summary of the relevant literature on the subject. Despite some attractive features of this scenario, it has been shown that it can only account for at most a few percent of all SNe Ia (Ilkov \& Soker 2013). Each of these formation channels has its own advantages and drawbacks, and in some cases these are severe. Currently, one of the most favored ones is the DD channel, because it provides adequate answers to two important observational facts, namely, the absence of hydrogen in the nebular phase (Leonard 2007) and the delay time distribution (Totani et al. 2008). Consequently, we focus on it.

Smoothed particle hydrodynamics (SPH) simulations of the coalescence of binary white dwarfs show that a prompt explosion is not always the result of the interaction during the dynamical phase of the merger (Yoon et al. 2007; LorénAguilar et al. 2009). Only those binary systems in which both the secondary and primary stars are massive enough lead to an SN Ia outburst. Actually, the parameter space for violent mergers is very narrow (Pakmor et al. 2010; Sato et al. 2015), 
and only when two carbon-oxygen white dwarfs of masses larger than $\sim 0.8 M_{\odot}$ merge is the result of the dynamical phase a prompt explosion. Within this theoretical framework, the dynamical phase of the merger is followed by a second phase in which the material of the debris region can be accreted and possibly lead to an SN Ia explosion.

The existing simulations of the coalescence of binary white dwarfs (Benz et al. 1990; Lorén-Aguilar et al. 2009; Raskin et al. 2012; Zhu et al. 2013; Dan et al. 2014) show that the final result of the coalescence consists of a central white dwarf made of the undisrupted primary star and a hot, convective corona made of about half of the mass of the disrupted secondary. This central remnant is surrounded by a heavy Keplerian disk, made of the rest of the mass of the disrupted secondary, since little mass is ejected from the system during the merger episode. The rapidly rotating, hot corona is convective, and an efficient $\alpha \omega$ dynamo can produce magnetic fields of up to $B \approx 10^{10} \mathrm{G}$ (García-Berro et al. 2012). Recent two-dimensional magnetohydrodynamic simulations of post-merger systems confirm the growth of the white dwarf magnetic field after the merger (Ji et al. 2013; Zhu et al. 2015). Thus, the role of magnetic fields in the aftermath of the dynamical merger needs to be explored. This is precisely the aim of the present work, as very few studies (Ji et al. 2013; Külebi et al. 2013; Beloborodov 2014) of the post-merger evolution including magnetism have been done so far. On the other hand, there are few works that explore the evolution of the post-merger systems but without considering the effects of the central remnant magnetic fieldsee, e.g., Yoon et al. (2007), van Kerkwijk et al. (2010), Shen et al. (2012), and Schwab et al. (2012).

Our paper is organized as follows. In Section 2 we explain the model of the evolution of the post-merger system. In particular, we describe the torques that act on the central remnant (Section 2.1), the structure of the rotating central white dwarf (Section 2.2), and its thermal evolution (Section 2.3). In Section 3 we present the prescriptions adopted to model the accretion rate on the central star. In Section 4 we present our choice of initial conditions. In Section 5 we discuss extensively the results of our simulations for two prescriptions to compute the accretion rate. Specifically, in Section 5.1 we discuss the case in which the accretion rate is obtained employing the cooling timescale, while in Section 5.2 we present the results obtained when it is computed using the viscous timescale. Finally, in Section 6 we compare our results with previous works, and in Section 7 we summarize our major findings, elaborate on their significance, and present our concluding remarks.

\section{Numerical Setup for the Post-merger Evolution}

Numerical simulations of binary white dwarf mergers indicate that in those cases in which a violent merger does not occur the merged configuration has three distinct regions (Benz et al. 1990; Guerrero et al. 2004; Lorén-Aguilar et al. 2009; Raskin et al. 2012; Zhu et al. 2013; Dan et al. 2014). First, there is the central white dwarf that rotates as a rigid solid. On top of it a hot, differentially rotating, convective corona can be found. This corona is made of matter accreted from the disrupted secondary star. The mass of this region is about half of the mass of the secondary white dwarf. Finally, surrounding these two regions there is a rapidly rotating Keplerian disk, which is made of the rest of the material of the disrupted secondary, since only a small amount of mass is ejected from the system during the coalescence. The evolution of the post-merger configuration depends, naturally, on the mass of the central remnant, which is made of the primary white dwarf and the hot corona, and on the properties of the surrounding disk.

Before entering into details, it is important to discuss some timescales, which are relevant to adopting a reasonable approximation for the evolution of the system. The postmerger configuration formed in the coalescence has a clear hierarchy of timescales. First comes the dynamical timescale $t_{\text {dyn }} \sim \Omega^{-1}$, with $\Omega$ being the rotation velocity. This timescale is typically of the order of a few seconds. Next in order of magnitude is the viscous timescale, $t_{\mathrm{visc}}$, of the Keplerian disk. This timescale governs the transport of disk mass inward and of angular momentum outward. The viscous phase of the evolution is normally followed using the Shakura-Sunyaev $\alpha$ prescription (Shakura \& Sunyaev 1973). Adopting typical values, van Kerkwijk et al. (2010) found that $t_{\text {visc }}$ ranges between $10^{3}$ and $10^{4} \mathrm{~s}$. However, for the merger remnants studied here we find that the viscous timescales could be significantly shorter, typically $1 \mathrm{~s}$-see Section 3.2. These viscous timescales are a little longer than, but of the same order as, the dynamical timescale of the post-merger, superChandrasekhar white dwarf studied here $\left(t_{\mathrm{dyn}} \sim 0.2-0.3 \mathrm{~s}\right.$; see Section 2.2). Finally, in this set of characteristic times is the thermal timescale of the merger product, $t_{\text {th }}$, which is typically much longer and will be discussed in detail for every simulation.

In the following we first discuss the interactions between the remnant and the disk in order to identify the torque that acts on the central remnant and model the evolution of its angular momentum. Then we present how we model the structure of the rotating white dwarf central remnant, as well as its thermal evolution. The fate of the white dwarf depends crucially on the accretion rate in the post-merger phase. Thus, we consider two very different physical scenarios to determine the accretion rate onto the central remnant. In the first of them the accretion rate is determined by the viscous timescale, whereas in the second scenario the accretion rate is governed by the thermal timescale. These will be discussed in Section 3.

\subsection{Torques on the Central Remnant}

The most commonly employed model of disk evolution is that of Ghosh \& Lamb (1979). This model was later improved by Wang (1987, 1995). Within this model the magnetic field of the remnant penetrates the disk in a broad transition zone as a result of the Kelvin-Helmholtz instabilities, turbulent diffusion, and magnetic field reconnection. Furthermore, the material of the disk corotates with the star only in a narrow region and reaches its surface channeled by the magnetic field lines. We assume that the magnetic field of the remnant penetrates the disk up to approximately the Alfvén radius (Davidson \& Ostriker 1973):

$$
R_{\mathrm{mag}}=\left(\frac{\mu_{\mathrm{WD}}^{2}}{\dot{M}_{\mathrm{disk}} \sqrt{2 G M_{\mathrm{WD}}}}\right)^{2 / 7},
$$

where $\mu_{\mathrm{WD}}=B_{\mathrm{WD}} R_{\mathrm{WD}}^{3}$ is the magnetic moment of the star, $B_{\mathrm{WD}}$ is its magnetic field, and $\dot{M}_{\text {disk }}$ is the mass flow through the inner radius of the disk, $R_{0}$.

The angular momentum per unit mass entering into the magnetosphere of the white dwarf through the inner radius is 
$l_{0}=R_{0}^{2} \Omega_{\mathrm{K}}^{0}$, where

$$
\Omega_{\mathrm{K}}^{0}=\left(\frac{G M_{\mathrm{WD}}}{R_{0}^{3}}\right)^{1 / 2}
$$

is the Keplerian angular velocity at $R_{0}$. This material is channeled by the magnetic field onto the surface of the remnant. Thus, the resulting spin-up torque on the star due to accretion of disk matter is given by

$$
T_{\mathrm{acc}}=\xi_{\mathrm{acc}} \dot{M}_{\mathrm{WD}} R_{0}^{2} \Omega_{\mathrm{K}}\left(R_{0}\right),
$$

where $\dot{M}_{\mathrm{WD}}=\varepsilon \dot{M}_{\text {disk }}$ is the mass accretion rate on the white dwarf, $\varepsilon$ measures how efficient accretion is, and $\xi_{\text {acc }}$ is a parameter that accounts for the deceleration of the accreted matter in the inner region of the disk. The values adopted for these two parameters will be discussed below. If the star rotates faster than the matter at the inner disk radius, the centrifugal barrier blocks this material. Hence, it cannot reach the surface of the newly born white dwarf. This matter is therefore ejected from the system. This happens when the inner edge of the disk moves beyond the corotation radius, which is beyond the distance at which the disk rotates with the same angular velocity of the central object:

$$
R_{\mathrm{co}}=\left(\frac{G M_{\mathrm{WD}}}{\Omega_{\mathrm{WD}}^{2}}\right)^{1 / 3} .
$$

When $R_{0}>R_{\mathrm{co}}$, the system enters into the dubbed propeller regime (Illarionov \& Sunyaev 1975). During this phase, the material reaching the magnetosphere is ejected with higher specific angular momentum than the one it had previously, thus resulting in a spin-down torque acting on the star. This torque is given by (Menou et al. 1999)

$$
T_{\text {prop }}=\sqrt{G M_{\mathrm{WD}} R_{0}} \dot{M}_{\text {disk }}\left(1-\omega_{\mathrm{f}}\right) .
$$

In this expression we have introduced the so-called fastness parameter:

$$
\omega_{\mathrm{f}}=\Omega_{\mathrm{WD}} / \Omega_{\mathrm{K}}\left(R_{0}\right)=\left(R_{0} / R_{\mathrm{co}}\right)^{3 / 2} .
$$

The rotating magnetic field of the star originates an induced electric field that results in a wind. The wind fills the magnetosphere and corotates with the star (Goldreich \& Julian 1969). At the light cylinder, $R_{\mathrm{lc}}=c / \Omega_{\mathrm{WD}}$, the corotation velocity reaches the speed of light, delimiting the region within which corotation of the magnetosphere is enforced. At larger distances, the field lines become open. For the spin-down torque due to electromagnetic energy losses in a force-free magnetosphere, $T_{\mathrm{dip}}$, we adopt the result of Spitkovsky (2006):

$$
T_{\text {dipole }}=k_{1} \frac{\mu_{\mathrm{WD}}^{2} \Omega_{\mathrm{WD}}^{3}}{c^{3}}\left(1+k_{2} \sin ^{2} \theta\right),
$$

where $k_{1}=1 \pm 0.05, k_{2}=1 \pm 0.1$, and $\theta$ is the angle between the magnetic moment and the rotation axis of the star.

Finally, an additional torque, $T_{\text {mag }}$, results from the interaction of the disk with the magnetic field of the white dwarf. According to Ghosh \& Lamb (1979), matter of the disk moving in the magnetic field of the white dwarf generates currents that confine the stellar field inside the disk. The magnetic field threading the disk is $\boldsymbol{B}_{\text {disk }}=\eta \boldsymbol{B}_{\mathrm{WD}}^{\mathrm{p}}$, where $\boldsymbol{B}_{\mathrm{WD}}$ is the magnetic field of the white dwarf, $\boldsymbol{B}_{\mathrm{WD}}^{\mathrm{p}}$ is its projection on the plane of the disk, and $\eta \leqslant 1$ is the screening coefficient that accounts for the effect of currents in the partially diamagnetic disk induced by the stellar field. In particular, the poloidal field induces an azimuthal current due to the radial motion of the plasma, which partially screens the stellar magnetic field. Also, the relative motion between the disk and the star magnetosphere generates a toroidal field, $B_{\phi}$. In the simulations, we have considered that the growth of $B_{\phi}$ is limited by diffusive decay due to turbulent mixing within the disk (Wang 1995). Following the analytical formulation of Wang (1997), the magnetic torque acting on the central star due to its interaction with the disk is given by

$$
\begin{aligned}
T_{\mathrm{mag}}= & \frac{\Gamma \eta^{2} \mu_{\mathrm{WD}}^{2}}{R_{0}^{3}} \\
& \times\left[\frac{2 h}{R_{0}}\left(1-\omega_{f}\right) \sin ^{2} \theta+\frac{\cos ^{2} \theta}{3}\left(1-2 \omega_{f}\right)\right],
\end{aligned}
$$

where $h \ll 1$ is the thickness of the disk and $\Gamma \simeq 1$ is a parameter that characterizes the steepness in the vertical transition from Keplerian rotation inside the disk to corotation with the star at the top of the disk. In the derivation of Equation (8), the magnetic field of the white dwarf has been approximated as a dipole, and it was assumed that the rotating axis of the star is perpendicular to the plane of the disk. Note that this expression generalizes the models of Wang (1987, 1995).

Based on this, the post-merger evolution of the angular momentum of the white dwarf is the result of the combined effect of the dipole radiation torque, $T_{\text {dipole, }}$, the accretion torque, $T_{\mathrm{acc}}$, the disk-interaction torque, $T_{\mathrm{disk}}$, and the propeller torque $T_{\text {prop }}$ :

$$
\dot{J}_{\mathrm{WD}}= \begin{cases}T_{\mathrm{acc}} & R_{\mathrm{WD}}>R_{0} \\ T_{\mathrm{acc}}+T_{\text {dipole }}+T_{\mathrm{mag}} & R_{\mathrm{WD}}<R_{0} \wedge \omega_{\mathrm{f}} \leqslant 1 . \\ T_{\text {dipole }}+T_{\mathrm{prop}} & R_{\mathrm{WD}}<R_{0} \wedge \omega_{\mathrm{f}}>1\end{cases}
$$

In the simulations presented here we assumed that the inner disk radius is the Alfvén radius.

\subsection{Rotating White Dwarf Configurations}

We follow the evolution of the spin of the post-merger remnant, calculating at every time step the new stable rotating configuration with mass, $M_{\mathrm{WD}}+\delta M$, and angular momentum, $J_{\mathrm{WD}}+\delta J$, adopting the slow rotation approximation (Hartle 1967; Hartle \& Thorne 1968). We also assumed that the central remnant product of the merger rotates as a rigid body, as predicted by detailed SPH simulations (Lorén-Aguilar et al. 2009). The equation of state of the white dwarf is assumed to be that of a zero-temperature degenerate electron gas (Chandrasekhar 1931), to which we add the corresponding contribution of ions.

The region of stability of uniformly rotating white dwarfs is bound by the secular axisymmetric instability limit, the mass shedding or Keplerian limit, and the inverse $\beta$-decay instability limit, which for pure carbon is $3.49 \times 10^{10} \mathrm{~g} \mathrm{~cm}^{3}$ (Rotondo et al. 2011; Boshkayev et al. 2013).

Figure 1 shows the mass-central density relation for the general relativistic uniformly rotating white dwarfs: the static, 


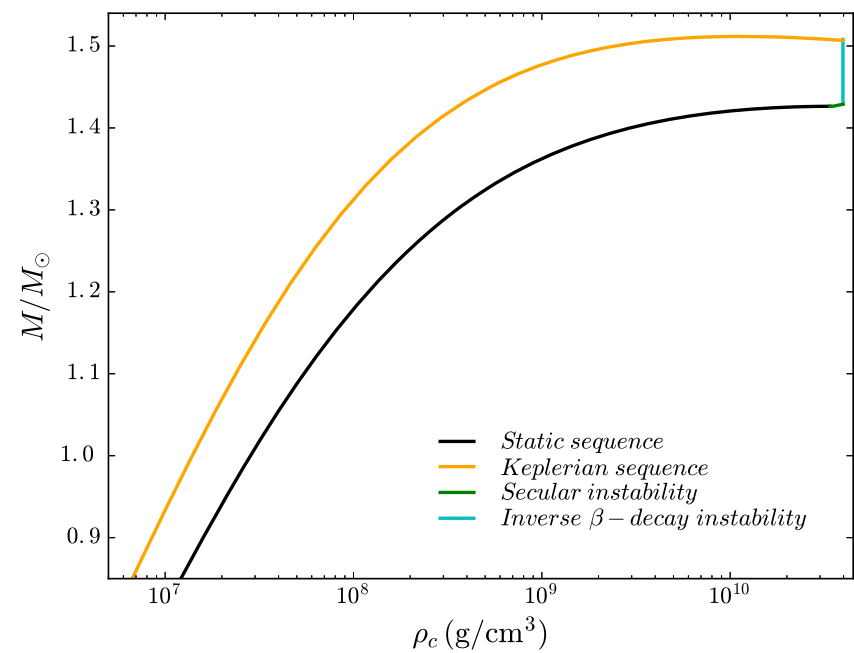

Figure 1. Instability lines of rotating white dwarfs in the diagram of mass vs. central density. The stability region is delimited by the static sequence (black line), the Keplerian sequence (orange line), the line of secular instability (green line), and the inverse $\beta$-decay line (blue).

Keplerian, secular axisymmetric instability and inverse $\beta$-decay sequences enclose the stability region. The mass-shedding, or Keplerian, limit is reached when the angular velocity of the star equals the Keplerian velocity of a particle orbiting at the equator, namely, when the centrifugal and gravitational forces are balanced. In this situation matter at the surface of the star is marginally bound to it. Hence, small perturbations result in mass loss until the star either becomes stable again or arrives at a dynamical instability point (Shibata et al. 2000; Stergioulas 2003). If the white dwarf crosses the $\beta$-decay instability limit, electrons are captured by nuclei. Since the principal contribution to the pressure of the star comes from electrons, electron captures reduce the pressure, leading to an instability (Salpeter 1961). The secular axisymmetric instability arises because the star is unstable with respect to axisymmetric perturbations. In a first phase, the star is expected to evolve quasi-stationarily with the instability growth timescale, which depends on the time required for angular momentum to be redistributed either by viscous dissipation or by the emission of gravitational waves (Chandrasekhar 1970). This timescale is typically long compared to the dynamical timescale (Boshkayev et al. 2013), except in the nonrotating case, where they are equal, because in this case there is no angular momentum to be redistributed. Eventually, when the star crosses the dynamical instability limit, gravitational collapse takes place (Stergioulas 2003). A sufficient (but not necessary) condition for the onset of secular instability can be obtained using the turning-point method (Friedman et al. 1988). This method considers a sequence of uniformly rotating models of a given (constant) angular momentum. The secular axisymmetric instability sets in at the density of the turning point:

$$
\left(\frac{\partial M\left(\rho_{c}, J\right)}{\partial \rho_{c}}\right)_{J}=0 .
$$

Finally, the maximum mass of static configurations is the Chandrasekhar limiting mass $M_{\max }=M_{\mathrm{Ch}} \approx 1.4 M_{\odot}$, while the maximum mass of rotating white dwarfs lies on the Keplerian sequence $M_{\max }^{J \neq 0}=1.52 M_{\odot}$. The configurations with mass between $M_{\max }^{J=0}<M_{\mathrm{WD}}<M_{\max }^{J \neq 0}$ are called super-Chandrasekhar white dwarfs and are metastable since they are supported by rotation. As the angular momentum of the star varies, the central white dwarf necessarily evolves toward one of the previously described instability limits (mass-shedding or secular axisymmetric instability).

\subsection{Temperature of the Central Remnant}

We turn now to the thermal evolution of the central white dwarf remnant. We have seen above in Sections 2.1 and 2.2, and we shall see below from the energy balance and transport equations, that the structure and thermal evolution of the white dwarf depend crucially on the accretion rate, which is set by the disk physics. In Section 3 we analyze the evolution and fate of the post-merger system for two physical prescriptions to set the accretion rate. In the first case the infall rate is driven by the disk angular momentum transport (viscous) timescale, which, owing to its shortness for the present binary parameters, leads to highly super-Eddington rates. It has been argued in the literature that the dissipation required to produce such very short viscous timescales might heat the disk to the point of carbon ignition (see, e.g., Mochkovitch \& Livio 1990). Such heating effects are not accounted for in the disk model adopted here. In addition, we also compute the evolution of the system in the case when the matter infall is driven not by the viscous timescale but by the cooling timescale. We show that this assumption leads to accretion rates near the Eddington limit value.

The thermal evolution of an accreting white dwarf has been studied by many authors (Nomoto \& Iben 1985; Saio \& Nomoto 1985; Yoon et al. 2007). The main goal of most of these works was to establish whether the conditions that may lead to an SN Ia were reached. For instance, in a pioneering work Nomoto \& Iben (1985) found that for accretion rates larger than a critical value $\dot{M}_{\text {crit }} \approx 2 \times 10^{-6} M_{\odot} \mathrm{yr}^{-1}$ the outer layers of the star are heated by mass accretion, while heat conduction and neutrino emission cool it. This results in a thermal inversion near the surface of the star and ultimately leads to an off-center carbon ignition. Saio \& Nomoto (1985) followed the subsequent evolution of the star and determined that carbon is burned in the entire white dwarf, being the final outcome of an oxygen-neon white dwarf. However, these works only considered nonrotating configurations and constant accretion rates. More recently, Yoon et al. (2007) studied the viscous evolution of remnants of white dwarf mergers, introducing the effects of rotation, but did not consider the effects of the magnetic field of the white dwarf. The postmerger object was modeled as a differentially rotating white dwarf with a cold core and a hot envelope accreting mass from a surrounding Keplerian disk at a constant rate. This allowed them to compute the conditions under which the off-center ignition can be avoided for the case in which the magnetic field is not taken into account.

We have explained above (see Section 2.1) how the rotation rate of the merged remnant evolves owing to the interaction of the magnetic field and the disk, which are the equilibrium configurations of the rotating white dwarf. However, during the course of the evolution the temperature of the white dwarf also changes owing to accretion from the disk, which heats the white dwarf interior. This, in turn, may trigger an $\mathrm{SN}$ Ia explosion. In order to assess this possibility, we follow the evolution of the interior temperature of the post-merger central white dwarf in an approximate way, which is described next. 
The equation of energy conservation reads

$$
\frac{d L}{d m}=\epsilon_{\mathrm{nuc}}-\epsilon_{\nu}+T \dot{s},
$$

where $m$ is the mass coordinate (i.e., the mass enclosed within the radial distance $r$ ), $L$ is the luminosity, $T$ is the temperature, $s$ is the specific entropy, and $\epsilon_{\text {nuc }}$ and $\epsilon_{\nu}$ are the energy release and energy loss per unit mass by nuclear reactions and neutrino emission, respectively.

For carbon-oxygen white dwarfs, thermonuclear energy is essentially released by two nuclear reactions:

$$
\begin{aligned}
& { }^{12} \mathrm{C}+{ }^{12} \mathrm{C} \rightarrow{ }^{20} \mathrm{Ne}+\alpha+4.62 \mathrm{MeV}, \\
& { }^{12} \mathrm{C}+{ }^{12} \mathrm{C} \rightarrow{ }^{23} \mathrm{Na}+\mathrm{p}+2.24 \mathrm{MeV},
\end{aligned}
$$

with nearly the same probability. We adopted the carbon fusion reaction rates of Gasques et al. (2005), which are valid for all regimes of $\rho$ and $T$, which is from the thermonuclear regime to the pynonuclear regime. For the neutrino energy losses, we used the analytical fits of Itoh et al. (1996), which consider electronpositron pair annihilation $\left(e^{-} e^{+} \rightarrow \nu \bar{\nu}\right)$, photo-neutrino emission $(e+\gamma \rightarrow e \nu \bar{\nu})$, plasmon decay $(\gamma \rightarrow \nu \bar{\nu})$, and electron-nucleus bremsstrahlung $[e(Z, A) \rightarrow e(Z, A) \nu \bar{\nu}]$. As will be discussed below, the dominant channel for neutrino losses in the central regions is electron-nucleus bremsstrahlung, while in the outer layers emission of neutrinos is dominated by plasmon decay.

The energy flux is given by

$$
\frac{d T}{d r}=-\frac{3}{16 \sigma} \frac{\kappa \rho}{T^{3}} \frac{L}{4 \pi r^{2}},
$$

where $\sigma$ is the Stephan-Boltzmann constant and $\kappa$ is the opacity, which can be written as

$$
\frac{1}{\kappa}=\frac{1}{\kappa_{\text {cond }}}+\frac{1}{\kappa_{\text {rad }}},
$$

with $\kappa_{\text {rad }}$ and $\kappa_{\text {cond }}$ the Rosseland mean radiative opacity and the conductive opacity, respectively. In the white dwarf core degeneracy is so high that the most efficient transport mechanism is conduction. Hence, the opacity is dominated by the first term. We adopted the thermal conductivity of Itoh et al. (1983, 1984), whereas for the radiative opacity we used Kramer's law, $\kappa_{\text {rad }}=4.34 \times 10^{24} \rho T^{-3.5} \mathrm{~cm}^{2} \mathrm{~g}^{-1}$.

The change of entropy with time can be obtained from

$$
T \dot{s}=c_{v} \dot{T}-\left[\frac{P}{\rho^{2}}-\left(\frac{\partial u}{\partial \rho}\right)_{T}\right] \dot{\rho},
$$

where $u$ is the specific internal energy and $c_{v}$ is the specific heat capacity at constant volume. The first term on the right-hand side of Equation (16) corresponds to the release of the internal energy, while the second term accounts for the change of gravitational potential energy due to the expansion or compression of the configuration.

To evaluate the term in square brackets in Equation (16), we considered a fully ionized nonideal electron-ion plasma, taking into account the ion-ion and the ion-electron Coulomb interactions, as well as the exchange-correlation corrections of electrons. We note that for super-Chandrasekhar white dwarfs both Coulomb corrections and quantum effects are important. The importance of Coulomb corrections is measured by the Coulomb coupling parameter $\Gamma=Z^{2} e^{2} /(a T)$, where $a=\left(3 /\left(4 \pi n_{i}\right)^{1 / 3}\right.$ is the mean interaction distance and $n_{i}$ is the ion number density. At $\Gamma \lesssim 1$ the ions behave as a gas, at $\Gamma>1$ as a strongly coupled Coulomb liquid, while crystallization occurs at $\Gamma \approx 175$. Quantum effects become important at temperatures smaller than $T_{\mathrm{p}}=\hbar \omega_{\mathrm{p}} / k_{\mathrm{B}}$, where $\omega_{\mathrm{p}}=$ $\left(4 \pi Z^{2} e^{2} n_{i} / m_{i}\right)^{1 / 2}$ is the ion plasma frequency. Finally, we used analytical fits to the heat capacity obtained from the free energy computed by Chabrier \& Potekhin (1998) and Potekhin \& Chabrier (2000).

Following Nomoto (1982), we obtained the density change by adopting the white dwarf mass coordinate, $q_{\mathrm{WD}} \equiv m / M_{\mathrm{WD}}$, as the independent variable:

$$
\dot{\rho}=\left[\left(\frac{\partial \rho}{\partial M_{\mathrm{WD}}}\right)_{q_{\mathrm{WD}}}-\frac{q_{\mathrm{WD}}}{M_{\mathrm{WD}}}\left(\frac{\partial \rho}{\partial q_{\mathrm{WD}}}\right)_{M_{\mathrm{WD}}}\right] \dot{M}_{\mathrm{WD}} .
$$

This equation explicitly provides the contribution of the global structural changes, as well as the contribution of the internal distribution of density to the thermal evolution during the accretion process.

The post-merger evolution of the system is computed assuming that the central white dwarf evolves in a sequence of stable configurations (see Section 2.2). To calculate the evolution of the temperature, at each time step we integrate Equations (11) and (14) using Equations (16) and (17).

In order to integrate Equations (11) and (14), a set of boundary conditions at the surface of the star must be adopted. We treat the material accreted in each interval time as a thin envelope surrounding the star. Following Townsley \& Bildsten (2004), we assume that the accreted material enters on top of the shell and pushes down the existing material deeper into the star. Then, the local heat equation becomes

$$
T\left(\frac{\partial}{\partial t}+v_{r} \frac{\partial}{\partial r}\right) s \approx T v_{r} \frac{\partial s}{\partial r}=\frac{d L}{d m}-\left(\epsilon_{\text {nuc }}-\epsilon_{\nu}\right),
$$

where $v_{r}=\dot{M}_{\mathrm{WD}} /\left(4 \pi r^{2} \rho\right)$ is the velocity of the material given by mass conservation. We constructed static envelopes for each stable configuration with total mass $M_{\mathrm{WD}}$ and radius $R_{\mathrm{WD}}$, integrating Equations (14) and (18).

To analyze whether the newly formed white dwarf reaches during its evolution the conditions suitable to produce an SN Ia, we adopted the following procedure. We first require as a necessary condition that the white dwarf crosses the ignition curve, i.e., the curve in the $\log \rho-\log T$ plane where the nuclear energy released becomes larger than the neutrino emissivity, $\epsilon_{\text {nuc }}=\epsilon_{\nu}$. For temperatures and densities beyond this curve nuclear energy production exceeds neutrino losses and the star is heated, possibly leading to an SN outburst. Additionally, we require that burning proceeds in an almost instantaneous way. The characteristic time $\tau_{\mathrm{CC}}$ of nuclear reactions is

$$
\tau_{\mathrm{CC}}=\frac{\epsilon_{\mathrm{nuc}}}{\dot{\epsilon}_{\mathrm{nuc}}} \approx \epsilon_{\mathrm{nuc}}\left(\dot{T} \frac{\partial \epsilon_{\mathrm{nuc}}}{\partial T}\right)^{-1}=c_{p}\left(\frac{\partial \epsilon_{\mathrm{nuc}}}{\partial T}\right)^{-1}
$$

where $c_{p}$ is the specific heat at constant pressure. If this characteristic timescale becomes shorter than the dynamical timescale

$$
\tau_{\text {dyn }}^{-1}=\sqrt{24 \pi G \rho},
$$


the star reaches the thermodynamic conditions necessary to explode as an SN Ia.

\section{Mass Accretion Rate on the Central Remnant}

As mentioned before, the accretion rate onto the post-merger central remnant can be computed in two different ways. Within the first approximation, the accretion rate is set by the thermal state of the envelope of the white dwarf (Yoon et al. 2007). Within the second one, the central remnant accretes mass from a thin Keplerian disk and its evolution is given by the viscous timescale-see van Kerkwijk et al. (2010), but also Ji et al. (2013). Since, due to the lack of full numerical analyses of this process with realistic physical ingredients, it is not yet clear which one of these treatments is more appropriate, we will calculate the evolution of the central white dwarf remnant adopting both prescriptions, and we will discuss the differences between both sets of calculations. In both cases we are interested in determining the long-term evolution central white dwarf taking into account the interaction between the disk and the magnetic field resulting from the merger. To handle this problem, we adopt a simplified picture of the post-merger system consisting of a magnetized rotating white dwarf surrounded by a thin Keplerian disk.

\subsection{Accretion Rate Set by the Thermal Timescale}

As discussed by Yoon et al. (2007), the assumption that the accretion timescale is set by the viscous timescale given by the Shakura-Sunyaev $\alpha$ prescription could be inappropriate to estimate the accretion timescale onto the newly formed white dwarf since the structure of the merged system deviates from a central, point-like mass surrounded by a thin disk that is the central assumption adopted within the $\alpha$-formalism. Yoon et al. (2007) argued that under these conditions the relevant timescale might be the cooling timescale of the low-density envelope between the central object and the disk. If the relevant timescale is the cooling timescale of the envelope of the postmerger white dwarf, the accretion rate will be given by

$$
\dot{M}_{\mathrm{WD}}=\frac{M_{\mathrm{disk}}}{\min \left(\tau_{\nu}, \tau_{\text {thermal }}\right)},
$$

where $M_{\text {disk }}$ is the total mass of the disk, and $\tau_{\nu}$ and $\tau_{\text {thermal }}$ are the neutrino cooling time and the thermal time on the envelope of the white dwarf, respectively. These two timescales are given by

$$
\tau_{\nu}=\left.\frac{c_{\mathrm{v}}}{\epsilon_{\nu}} T_{S}\right|_{S}
$$

and

$$
\tau_{\text {thermal }}=\frac{3}{64 \sigma}\left(\int_{\Delta r}\left(\frac{\kappa c_{v}}{T^{3}}\right)^{1 / 2} \rho d r\right)^{2}
$$

(Henyey \& L'Ecuyer 1969; Piersanti et al. 2003), where $c_{v}$ is the heat capacity at constant volume, $\kappa$ is the opacity, $\epsilon_{\nu}$ is the energy release by the neutrino emission, and $\Delta r$ delimits the region of interest-see Section 2.3 for a discussion of the thermal properties of the white dwarf.

For the values typical of the post-merger white dwarf ( $\rho=10^{6} \mathrm{~g} \mathrm{~cm}^{-3}$ and $T=10^{8} \mathrm{~K}$ ), the neutrino luminosity will be $L_{\nu} \sim 10^{2} L_{\odot}$ and the thermal energy will be $U \sim 10^{48} \mathrm{erg}$.
At the beginning of the simulations the neutrino cooling timescale is about $\tau_{\nu} \sim 6 \times 10^{5} \mathrm{yr}$, while the thermal timescale is $\tau_{\text {thermal }} \sim 10^{6} \mathrm{yr}$. Then, the initial accretion rate is about $\dot{M}_{\mathrm{WD}} \sim 10^{-7} M_{\odot} \mathrm{yr}^{-1}$, close to the Eddington limit.

\subsection{Accretion Rate Set by the Viscous Timescale}

Numerical simulations_-see, e.g., Benz et al. (1990), LorénAguilar et al. (2009), and Dan et al. (2011)—show that the disk product of the coalescence of two white dwarfs of unequal masses is not thin, although it is not thick either. Specifically, Lorén-Aguilar et al. (2009) found that the thickness of the newly formed disk is $H \sim 5.0 \times 10^{-3} R_{\odot}$, while the typical size is $R_{\text {disk }} \sim 0.2 R_{\odot}$-see their Table 1 . Hence, $H / R_{\text {disk }} \simeq$ 0.025 . Thus, assuming that the disk is thin is not an extreme assumption, and in the worst of the situations, it can be considered as a limiting case. Obviously, the other limiting case is to assume that the disk is thick. Here we assume that the disk is thin, and we postpone the study of a thick disk to a forthcoming publication.

For a thin, Keplerian accretion disk, the evolution of the surface mass density, $\Sigma$, is given by the diffusion equation:

$$
\frac{\partial \Sigma}{\partial t}=\frac{3}{r} \frac{\partial}{\partial r}\left[r^{1 / 2} \frac{\partial}{\partial r}\left(\nu \Sigma r^{1 / 2}\right)\right]
$$

where $\nu$ is the turbulent kinematic viscosity. To describe the time evolution of the disk, we use one of the three self-similar solutions of Equation (24) found by Pringle (1974). These solutions are obtained for an opacity parameterized as $\kappa=$ $\kappa_{0} \rho^{a} T^{b}$. Within this approximation mass is accreted onto the central white dwarf, but the angular momentum of the disk is conserved, $J_{\text {disk }}=J_{0}$, because the outer edge of the disk moves outward-see Equation (26). In a general case $J_{\text {disk }}$ would not be conserved because there is a trade-off of angular moment between the disk and the central white dwarf. However, since the angular moment of the white dwarf, $J_{\mathrm{WD}}$, is much smaller than that of the disk, this solution is accurate enough for the purpose of estimating the accretion rate onto the white dwarf. Actually, we have checked that at all times $J_{\mathrm{WD}} / J_{\text {disk }}<0.1$ in our simulations.

Within this approximation mass flows at the inner disk boundary at a rate

$$
\dot{M}_{\text {disk }}=\frac{(\beta-1) M_{0}}{t_{\text {visc }}}\left(1+\frac{t}{t_{\text {visc }}}\right)^{-\beta},
$$

and the disk outer radius evolves as

$$
R_{\mathrm{out}}=r_{0}\left(1+\frac{t}{t_{\mathrm{visc}}}\right)^{2 \beta},
$$

where $M_{0}$ and $J_{0}$ are, respectively, the initial mass and angular momentum of the disk, $j_{0}=J_{0} / M_{0}, r_{0}=3.88 j_{0}^{2} /\left(G M_{0}\right)$, and $\beta$ is a constant that depends on the opacity regime. Here we have adopted a bound-free opacity $(\beta=5 / 4)$. The viscous timescale within this model is determined by $M_{0}$ and $j_{0}$ as follows (Ertan et al. 2009):

$$
t_{\mathrm{visc}}=9.82 \times 10^{9} \frac{j_{0}^{25 / 7}}{M_{\mathrm{WD}}^{10 / 7} M_{0}^{3 / 7}}\left(\frac{\sigma}{\alpha^{8} \kappa_{0}}\right)^{1 / 7}\left(\frac{\bar{\mu} m_{p}}{\kappa_{B}}\right)^{15 / 14},
$$


Table 1

Parameters of the Simulations of Post-merger Remnants

\begin{tabular}{|c|c|c|c|c|c|c|c|c|c|c|c|c|c|}
\hline Model & $\begin{array}{c}M_{1} \\
\left(M_{\odot}\right)\end{array}$ & $\begin{array}{c}M_{2} \\
\left(M_{\odot}\right)\end{array}$ & $\begin{array}{l}M_{\mathrm{WD}} \\
\left(M_{\odot}\right)\end{array}$ & $\begin{array}{c}M_{0} \\
\left(M_{\odot}\right)\end{array}$ & $\begin{array}{l}R_{\mathrm{eq}, W D} \\
\quad\left(R_{\odot}\right)\end{array}$ & $\begin{array}{c}B_{\mathrm{WD}} \\
(\mathrm{G})\end{array}$ & $\begin{array}{c}\Omega_{\mathrm{WD}} \\
\left(\operatorname{rad~s}^{-1}\right)\end{array}$ & $\begin{array}{c}J_{\mathrm{WD}} \\
\left(\mathrm{g} \mathrm{cm}^{2} \mathrm{~s}^{-1}\right)\end{array}$ & $\begin{array}{c}J_{0} \\
\left(\mathrm{~g} \mathrm{~cm}^{2} \mathrm{~s}^{-1}\right)\end{array}$ & $\begin{array}{c}\varepsilon \\
(-)\end{array}$ & $\begin{array}{l}t_{\mathrm{dyn}} \\
(\mathrm{s})\end{array}$ & $\begin{array}{c}t_{\text {visc }} \\
(\mathrm{s})\end{array}$ & $\begin{array}{l}t_{\mathrm{cool}} \\
(\mathrm{yr})\end{array}$ \\
\hline A & 1.12 & 0.78 & 1.45 & 0.45 & 0.0026 & $10^{9}$ & 3.00 & $2.79 \times 10^{49}$ & $5.86 \times 10^{50}$ & 0.1 & 0.3 & 0.745 & $2.26 \times 10^{4}$ \\
\hline B & 1.12 & 0.78 & 1.45 & 0.45 & 0.0026 & $10^{6}$ & 3.00 & $2.79 \times 10^{49}$ & $5.86 \times 10^{50}$ & 0.1 & 0.3 & 0.745 & $2.26 \times 10^{4}$ \\
\hline $\mathrm{C}$ & 1.12 & 0.78 & 1.45 & 0.45 & 0.0026 & $10^{7}$ & 3.00 & $2.79 \times 10^{49}$ & $5.86 \times 10^{50}$ & 0.1 & 0.3 & 0.745 & $2.26 \times 10^{4}$ \\
\hline $\mathrm{D}$ & 1.12 & 0.78 & 1.45 & 0.45 & 0.0026 & $10^{8}$ & 3.00 & $2.79 \times 10^{49}$ & $5.86 \times 10^{50}$ & 0.1 & 0.3 & 0.745 & $2.26 \times 10^{4}$ \\
\hline $\mathrm{E}$ & 1.12 & 0.78 & 1.45 & 0.45 & 0.0026 & $10^{9}$ & 3.00 & $2.79 \times 10^{49}$ & $5.86 \times 10^{50}$ & 0.1 & 0.3 & 0.745 & $2.26 \times 10^{4}$ \\
\hline $\mathrm{F}$ & 1.12 & 0.78 & 1.45 & 0.65 & 0.0026 & $10^{9}$ & 3.00 & $2.79 \times 10^{49}$ & $5.86 \times 10^{50}$ & 0.1 & 0.3 & 0.253 & $2.26 \times 10^{4}$ \\
\hline G & 1.12 & 0.78 & 1.45 & 0.45 & 0.0026 & $10^{6}$ & 3.00 & $2.79 \times 10^{49}$ & $5.86 \times 10^{50}$ & 0.1 & 0.3 & 0.745 & $2.26 \times 10^{4}$ \\
\hline $\mathrm{H}$ & 1.12 & 0.78 & 1.45 & 0.45 & 0.0026 & $10^{9}$ & 3.00 & $2.79 \times 10^{49}$ & $5.86 \times 10^{50}$ & 0.5 & 0.3 & 0.745 & $2.26 \times 10^{4}$ \\
\hline I & 1.12 & 0.78 & 1.45 & 0.45 & 0.0039 & $10^{9}$ & 2.30 & $4.56 \times 10^{49}$ & $5.69 \times 10^{50}$ & 0.1 & 0.4 & 0.668 & $2.79 \times 10^{3}$ \\
\hline $\mathrm{J}$ & 1.12 & 0.78 & 1.45 & 0.45 & 0.0034 & $10^{9}$ & 2.50 & $3.87 \times 10^{49}$ & $5.76 \times 10^{50}$ & 0.1 & 0.4 & 0.697 & $5.66 \times 10^{3}$ \\
\hline $\mathrm{K}$ & 1.12 & 0.78 & 1.45 & 0.45 & 0.0032 & $10^{9}$ & 2.60 & $3.60 \times 10^{49}$ & $5.78 \times 10^{50}$ & 0.1 & 0.4 & 0.709 & $7.79 \times 10^{3}$ \\
\hline $\mathrm{L}$ & 1.12 & 0.78 & 1.45 & 0.45 & 0.0030 & $10^{9}$ & 2.70 & $3.35 \times 10^{49}$ & $5.83 \times 10^{50}$ & 0.1 & 0.4 & 0.719 & $1.05 \times 10^{4}$ \\
\hline M & 1.12 & 0.78 & 1.45 & 0.45 & 0.0029 & $10^{9}$ & 2.80 & $3.14 \times 10^{49}$ & $5.68 \times 10^{50}$ & 0.1 & 0.4 & 0.729 & $1.38 \times 10^{4}$ \\
\hline $\mathrm{N}$ & 1.12 & 0.78 & 1.45 & 0.45 & 0.0028 & $10^{9}$ & 2.85 & $2.93 \times 10^{49}$ & $5.68 \times 10^{50}$ & 0.1 & 0.4 & 0.665 & $1.57 \times 10^{4}$ \\
\hline $\mathrm{O}$ & 1.12 & 0.78 & 1.45 & 0.45 & 0.0028 & $10^{9}$ & 2.90 & $2.93 \times 10^{49}$ & $5.68 \times 10^{50}$ & 0.1 & 0.3 & 0.737 & $1.78 \times 10^{4}$ \\
\hline $\mathrm{P}$ & 1.12 & 0.78 & 1.45 & 0.45 & 0.0022 & $10^{9}$ & 3.50 & $2.24 \times 10^{49}$ & $5.86 \times 10^{50}$ & 0.1 & 0.3 & 0.769 & $5.34 \times 10^{4}$ \\
\hline Q & 1.12 & 0.78 & 1.45 & 0.45 & 0.0017 & $10^{9}$ & 4.50 & $1.75 \times 10^{49}$ & $5.96 \times 10^{50}$ & 0.1 & 0.2 & 0.793 & $8.79 \times 10^{4}$ \\
\hline
\end{tabular}

where $m_{p}$ is the proton mass and $\mu$ the mean molecular weight. Adopting $\alpha=0.1$ for the viscosity parameter (Shakura \& Sunyaev 1973) gives

$$
t_{\mathrm{visc}} \simeq 10.9\left(\frac{j_{0}}{10^{18} \mathrm{~cm}^{2} \mathrm{~s}^{-1}}\right)^{25 / 7}\left(\frac{M_{0}}{0.1 M_{\odot}}\right)^{-3 / 7} \mathrm{~s}
$$

This solution has been employed to describe debris disks around massive black holes formed by tidal disruption of stars (Cannizzo et al. 1990) and in SN fallback disks around young neutron stars (Chatterjee et al. 2000). This formulation was also used in Külebi et al. (2013) to study the long-term evolution of the disk interaction of magnetized white dwarfs resulting from white dwarf mergers that do not develop prompt explosion conditions.

We obtain that the initial conditions of the merged system are such that $t_{\text {visc }} \sim 0.2-0.8 \mathrm{~s}$ (see Table 1). This, in turn, results in accretion rates at early times $\dot{M}_{\mathrm{WD}}=M_{0} / t_{\mathrm{visc}} \sim 10^{-1} M_{\odot} \mathrm{s}^{-1}$ and an initial disk outer radius $R_{\text {out }} \sim 0.1 R_{\odot}$. However, for times longer than $t_{\mathrm{visc}}$, the accretion rate drops one order of magnitude, and after $10^{2} \mathrm{~s}$, the accretion is $10^{-4} M_{\odot} \mathrm{s}^{-1}$.

In the above equations the disk viscous timescale, $t_{\mathrm{visc}}$, is a constant. It is clear from Equation (27) that it is a good approximation for low infall rates because the specific angular momentum would not change appreciably. The above estimate of the accretion rate shows that this is not the present case, so we have checked the effect of the assumption of the constancy of $t_{\text {visc }}$ on the evaluation of $\dot{M}_{\text {disk }}$ as follows. First, we compute the accretion rate using Equation (25), keeping $t_{\text {visc }}$ constant and then allowing it to increase with time owing to the mass loss by the disk. We found that the increase of $t_{\text {visc }}$ would lower $\dot{M}_{\text {disk }}$ at most by $10 \%$ during the evolution. This result assures us about our assumption of a constancy of the viscous timescale on the estimate of the infall rate at the inner disk radius.

\section{Initial Conditions}

We compute the post-merger initial configuration assuming that both mass and angular momentum are conserved during the merger. This is a reasonable assumption, since SPH simulations show that very little mass is ejected from the system. Moreover, little angular momentum is carried away by the unbound material, since its velocity is mostly radial. A rough estimate of the degree to which mass and angular momentum are conserved is $J_{\mathrm{ej}} / J \sim M_{\mathrm{ej}} / M \sim 10^{-3}$ (LorénAguilar et al. 2009; Dan et al. 2014). Consequently, the orbital angular momentum of the coalescing white dwarfs is invested in spinning up the primary white dwarf and in the angular momentum of the rapidly rotating disk.

If the spin angular momentum of the merging white dwarfs is neglected, the total angular momentum just before the merger is

$$
J_{\mathrm{sys}}=q \sqrt{\frac{G M_{2}^{3}\left(R_{1}+R_{2}\right)}{(1+q)}},
$$

where $\left(M_{1}, R_{1}\right)$ and $\left(M_{2}, R_{2}\right)$ are the masses and radii of the merging stars, respectively, and $q=M_{2} / M_{1}$ is the mass ratio of the original binary system. Figure 2 shows the contours of constant angular momentum and of constant total mass in the plane defined by $M_{1}$ and $M_{2}$. Given an initial total mass and angular momentum of the remnant $\left(M_{\mathrm{WD}}, J_{\mathrm{WD}}\right)$ and an initial disk mass $M_{0}$, we computed the initial angular momentum of the disk, $J_{0}$, assuming that the central remnant rotates as a rigid body. To do this, we considered that just before the mass transfer episode the orbital separation was such that the secondary was about to fill its Roche lobe, for which we adopted the expression of Eggleton (1983), which is in all the cases close to $R_{1}+R_{2}$. This is the same as saying that the merger episode begins when both stars are in contact. We also took into account that according to detailed SPH simulations of the merger process roughly half of the mass of the secondary 


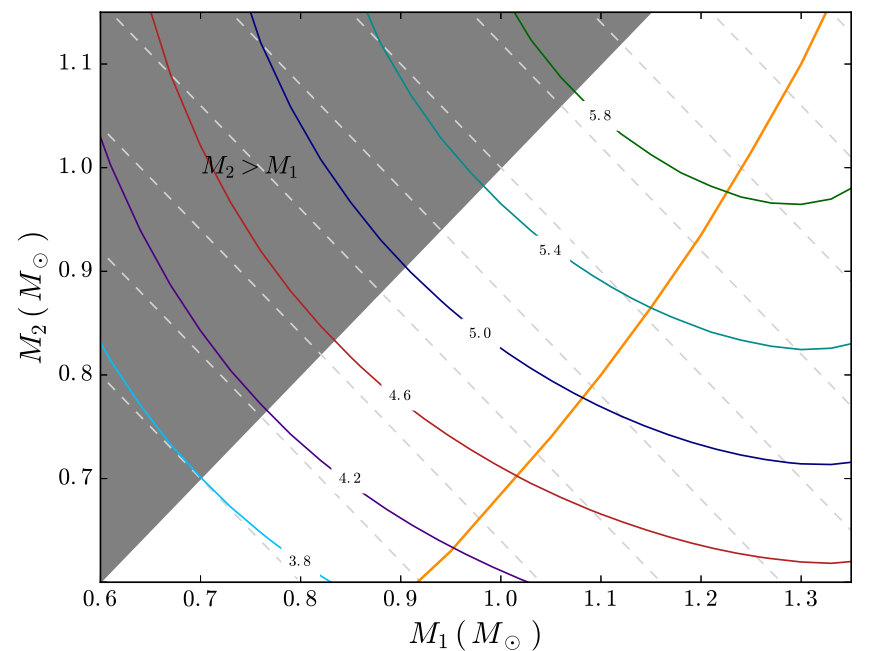

Figure 2. Parameter space of the initial white dwarf binary system. Solid lines are contours of constant total angular momentum, labeled in units of $10^{50} \mathrm{~g} \mathrm{~cm}^{2} \mathrm{~s}^{-1}$. The gray dotted straight lines are contours of constant total mass, from 1.4 to $2.6 M_{\odot}$, in steps of $0.1 M_{\odot}$. The shaded area corresponds to configuration with $q>1$. The orange line corresponds to the location of the system when the secondary is filling its Roche lobe $\left(R_{2}=R_{\mathrm{L}}\right)$.

star goes to form the disk, whereas the rest of the material is directly accreted onto the primary component of the binary system (Lorén-Aguilar et al. 2009).

Nevertheless, given the exploratory nature of the simulations presented here, we did not constrain ourselves to the values obtained in the SPH simulations, and we adopted different values for the mass of the remnant and for the mass of the disk. Since a rigidly rotating white dwarf can support a maximum angular momentum $\lesssim 10^{50} \mathrm{~g} \mathrm{~cm}^{2} \mathrm{~s}^{-1}$ (Boshkayev et al. 2013), angular momentum conservation implies that a substantial fraction of the angular momentum of the binary system goes to the Keplerian disk.

Also, we mention that detailed SPH simulations of nonsymmetric white dwarf mergers show that as the less massive white dwarf is disrupted and part of its matter is accreted onto the primary star, the accreted mass is compressed and heated. Thus, for all the cases studied here we have assumed an inverse initial temperature profile. Specifically, we adopted initial temperature profiles similar to those resulting from detailed SPH simulations.

In Table 1 we list the initial masses of the stars of the original binary system-Columns (2) and (3), respectively - as well as the initial conditions for the models presented in this work. The top section of this table lists the characteristics of the models for which the accretion rate was computed employing the cooling timescale, while the bottom section summarizes the most relevant information of those models computed employing the accretion rate given by the viscous timescale. Note that the mass of the rapidly rotating central white dwarf $M_{\mathrm{WD}}$ of all these simulations (listed in Column (4)) is the same. Since we are modeling white dwarfs as rigidly rotating configurations, only two parameters are needed to determine the remaining characteristics of the merged configuration. Thus, we decided to vary the initial angular velocity $\Omega_{\mathrm{WD}}$ and the mass of the disk $M_{0}$. These two quantities are listed in Columns (8) and (5), respectively. For a super-Chandrasekhar white dwarf of $1.45 M_{\odot}$, the minimum angular velocity for the gravitational stability to set in is around $2.03 \mathrm{rad} \mathrm{s}^{-1}$. Note that the angular velocities adopted here are in all cases larger than this value. Once the angular velocity of the white dwarf is known, we determine its total angular momentum, $J_{\mathrm{WD}}$, which is listed in Column (9) of the table, and the equatorial radius of the white dwarf, $R_{\mathrm{WD}}$, listed in Column (6). The adopted magnetic field $B_{\mathrm{WD}}$ of the white dwarf is given in Column (7). The rest of the columns of Table 1 list the initial angular momentum $J_{0}$ (Column (10)) of the disk and the efficient parameter $\varepsilon$ (Column (11)), which is relevant for estimating the mass accretion rate, the dynamical timescale $t_{\mathrm{dyn}}$, the viscous timescale $t_{\mathrm{visc}}$, and the cooling timescale of the disk $t_{\text {cool }}$ (Columns (12)-(14)).

In our simulations the magnetic field of the remnant was kept fixed at $B_{\mathrm{WD}}=10^{9} \mathrm{G}$, a representative value of the magnetic field resulting from the stellar dynamo that originated during the coalescence (García-Berro et al. 2012). For the typical values found in the SPH simulations of merging white dwarfs the magnetic field can be as high as $\sim 10^{10} \mathrm{G}$, depending on whether the dynamo is saturated. However, we decided to adopt a conservative value as a reference magnetic field, $10^{9} \mathrm{G}$. However, we also computed some models with various magnetic field strengths, ranging from $10^{6}$ to $10^{9} \mathrm{G}$. An important point that is worth emphasizing is that for the simulations presented here we assumed that during the merging process an ordered global dipole field is produced. However, this is not guaranteed-see, for instance, the discussion in the conclusions of Schwab et al. (2012).

\section{Results}

Since the evolution of the post-merger system depends critically on the adopted prescription for the mass accretion rate onto the central white dwarf resulting from the coalescence of the binary system, we discuss the results obtained using the accretion rates described in Sections 3.2 and 3.1 separately. We first discuss the results obtained using an accretion rate set by the cooling timescale, and subsequently we present the results obtained when the viscous timescale is employed.

\subsection{Accretion Rate Set by the Cooling Timescale}

To start with, we discuss our reference model. This is model A in Table 1. For this model we adopted a magnetic field $B=10^{9} \mathrm{G}$. We note that during the early phases of the evolution of the post-merger remnant the dominant cooling mechanism of the external layers of the central white dwarf is neutrino emission. Therefore, the initial relevant timescale to compute the accretion rate is $\dot{M}_{\mathrm{WD}}=M_{\mathrm{WD}} / \tau_{\nu} \sim 10^{20} \mathrm{~g} \mathrm{~s}^{-1}$. This accretion rate is of the order of the Eddington limit. However, in our calculations the evolution of the model is followed self-consistently. That is, we computed the mass accretion rate with the cooling timescale provided by the evolution-see below.

For this model, the magnetospheric radius is $R_{\mathrm{mag}}=0.062 R_{\odot}$ and the corotation radius is $R_{\mathrm{co}}=0.0039 R_{\odot}$. Thus, $R_{\mathrm{mag}}>$ $R_{\mathrm{co}}>R_{\mathrm{WD}}$, so the dipole radiation torque and the propeller torque drive the evolution of the spin of the remnant. Under these conditions the central object does not accrete matter. To illustrate the evolution, Figure 3 shows some temperature profiles at selected times. In particular, we show these profiles at times $t_{0}$ (corresponding to the beginning of the evolution; solid line) at a time just before the central white dwarf reaches explosive conditions (time $t=t_{3}$; long-dashed line) and at two intermediate stages (times $t_{1}$ and $t_{2}$; short-dashed line and dot-dashed line, respectively). As can be seen in this figure, during the entire 


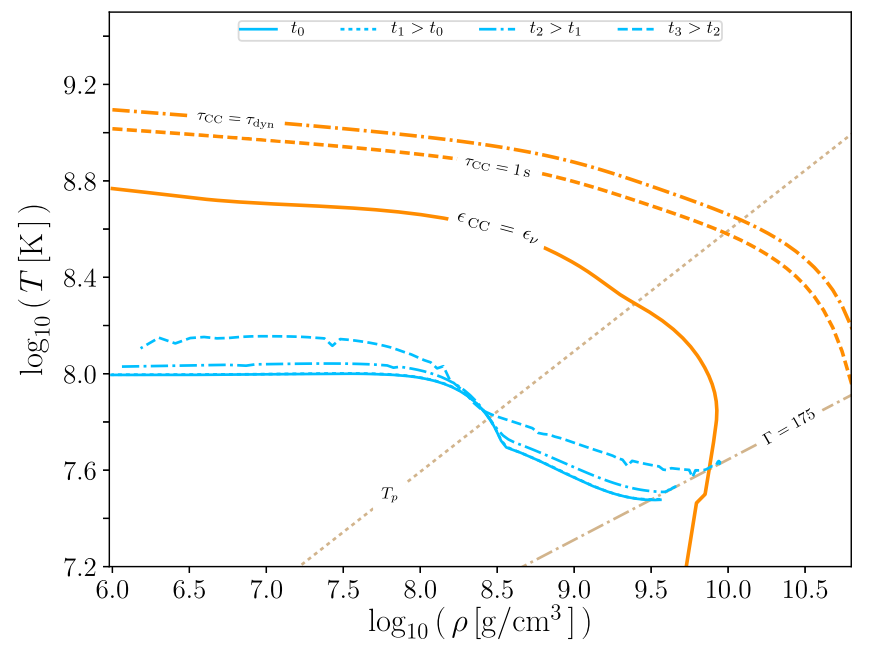

Figure 3. Temperature-density profiles of the central white dwarf at different times of the evolution for model A. This model was computed assuming $B=10^{9} \mathrm{G}$ and an accretion rate set by the cooling timescale. We also show the carbon ignition line, labeled as $\epsilon_{\mathrm{CC}}=\epsilon_{\nu}$, and two carbon-burning timescales $\tau_{\mathrm{CC}}=\tau_{\mathrm{dyn}}$ and $\tau_{\mathrm{CC}}=1 \mathrm{~s}$. In these panels the crystallization curve is labeled as $\Gamma=175$ and the plasma temperature as $T_{\mathrm{p}}$. The configuration at $t_{1}$ has a temperature profile almost equal to the initial one. At $t_{2}$ and $t_{3}$, it heats at the center and at the surface.

evolution both the central regions and the outer layers of the white dwarf are compressed and heated. These two regions of the white dwarf are separated by the line at which the temperature of the nearly isothermal core equals the plasma temperature, $T_{\mathrm{P}}$. This behavior is a consequence of the torques acting on the white dwarf. The acting torques brake the white dwarf, decreasing its angular velocity. As a consequence, the centrifugal force decreases and the gravitational force dominates. Thus, to balance the enhanced gravity, the density increases, as does the temperature. Ultimately, the center of the star reaches the thermodynamic conditions needed to burn carbon explosively. These conditions are illustrated in this figure by the curves $\epsilon_{\text {nuc }}=\epsilon_{\nu}, \tau_{\mathrm{CC}}=\tau_{\text {dyn }}$, and $\tau_{\mathrm{CC}}=1 \mathrm{~s}$. This occurs at time $40.8 \mathrm{yr}$, just before these regions reach the beta-decay instability limit, and when the central regions of the star are already crystallizing. Figure 4 displays the evolution of model A in the mass-central density plane. As can be seen, the evolutionary track corresponds to pure compression, and no matter is accreted. Note as well that carbon in the central regions of the star is ignited before the inverse $\beta$-decay instability is reached.

Figure 5 shows the run of the neutrino and thermal timescales in the white dwarf interior of model A at two relevant times, namely, $t=t_{0}$ and $t=t_{3}$, as previously defined. As can be seen, for $t=t_{0}$ neutrinos cool the external layers of the star, while in the internal regions thermal diffusion is the dominant transport mechanism. At time $t_{3}$ the relevant cooling timescale in the outer layers of the white dwarf is no longer the neutrino timescale, but the thermal timescale. Actually, it is important to realize that in the dense inner core of the white dwarf the shorter timescale is always the thermal timescale. Thus, Figure 5 clearly shows that the cooling timescale must be computed self-consistently along the evolution of the remnant to obtain physically sound results. We emphasize that although this plot illustrates these timescales for model $\mathrm{A}$, our calculations demonstrate that this is a representative case and that for all the models listed in the top section of Table 1 the run of these timescales is similar.

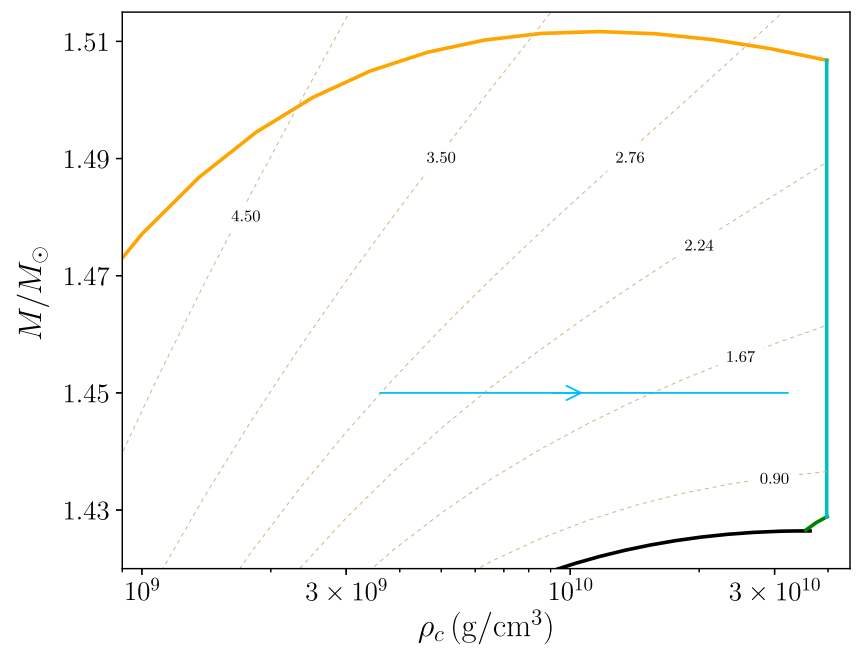

Figure 4. Evolution of model $\mathrm{A}$ in the mass-central density plane, at selected times; see text for details. The colors of the lines indicating the regions of the various instabilities discussed here are the same as shown in Figure 1. Also shown are the contours of constant angular momentum (dotted lines).

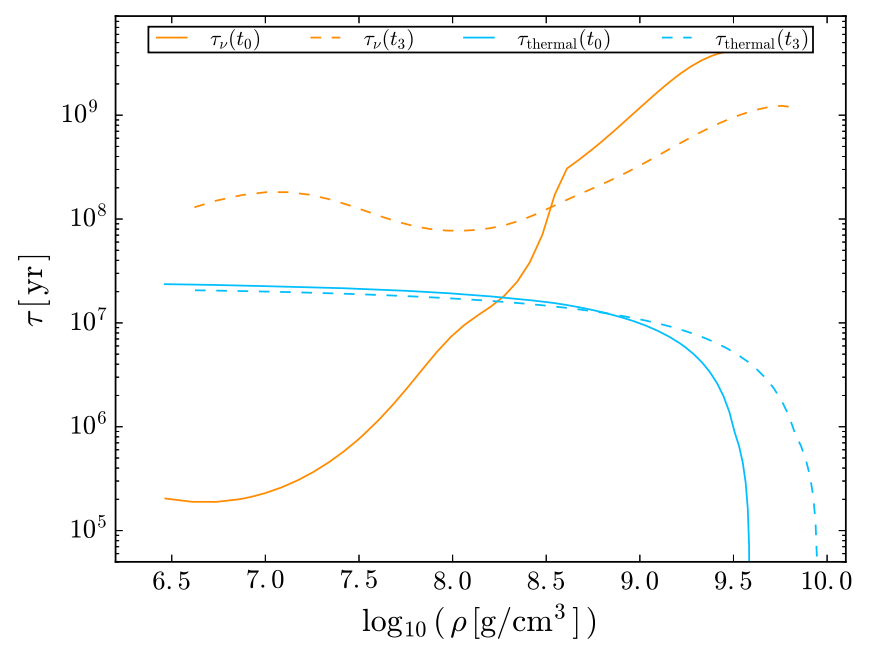

Figure 5. Run of the neutrino and thermal timescales in the interior of model A at selected times; see text for details.

To study the dependence of the evolution of the merged configuration on the magnetic field, we ran a suite of models with varying strengths of the magnetic field. These are models $\mathrm{B}, \mathrm{C}$, and D in Table 1, for which we adopted magnetic field strengths $B=10^{6}, 10^{7}$, and $10^{8} \mathrm{G}$, respectively, keeping unchanged the rest of the parameters of model A. For the sake of conciseness we only discuss model $\mathrm{B}$, which corresponds to the smallest magnetic field strength, $10^{6} \mathrm{G}$.

Figure 6 shows the evolution of the remnant at different times for this model. These times were selected using the same criteria we used previously, and the line coding is the same. In this case $R_{\mathrm{mag}}<R_{\mathrm{WD}}$ and the central white dwarf can accrete matter from the disk. Under these conditions we find that accretion onto the white dwarf heats the outer layers of the star, while the temperature of its core remains almost unchanged. Ultimately the very outer layers of the white dwarf are heated to such an extent that carbon is ignited off-center. However, an inspection of Figure 7, which shows the evolution in the masscentral density plane, reveals that actually the central regions of the star expand slightly. This again is due to the acting torques, which spin up the white dwarf. 


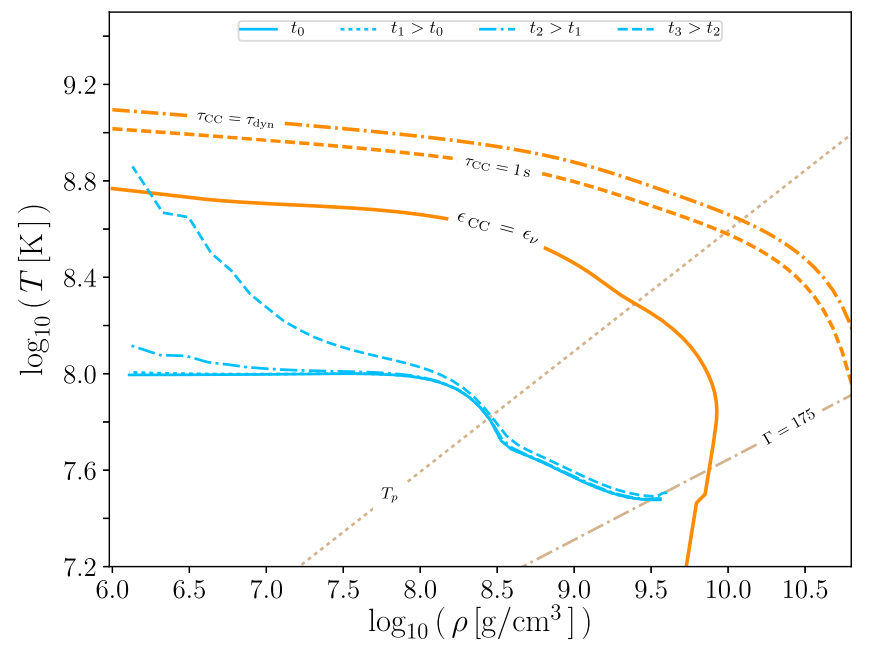

Figure 6. Same as Figure 3, but for the case in which a modest magnetic field $B=10^{6} \mathrm{G}$, is adopted. This is model $\mathrm{B}$ in Table 1 .

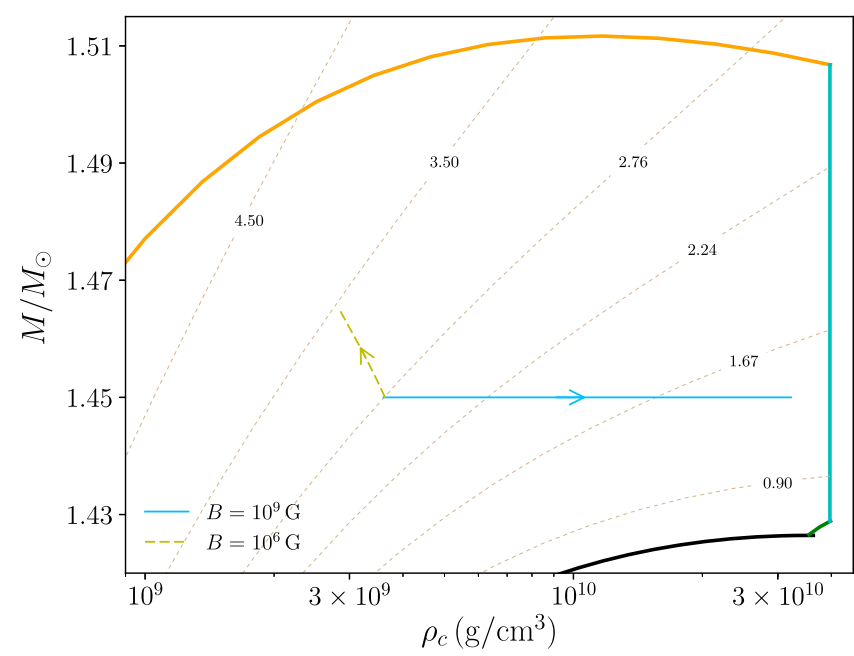

Figure 7. Same as Figure 4, but including the case in which a magnetic field, $B=10^{6} \mathrm{G}$, is adopted (yellow line). This is model B in Table 1 .

We found that if the magnetic field of the white dwarf is higher than $3.84 \times 10^{6} \mathrm{G}$, the magnetospheric radius is larger than the corotation radius. Thus, in this case the central remnant does not accrete more material after the merger, so the propeller torque and the dipole radiation torque drive the evolution of its spin rate. On the other hand, if the magnetic field is lower than $1.88 \times 10^{6} \mathrm{G}$, the magnetospheric radius is smaller than the white dwarf radius. Hence, the central white dwarf accretes material. Consequently, in this case the evolution of the rotation rate is driven by the accretion torque. Thus, for these initial conditions the magnetic field determines the accretion rate and the evolution of the star.

To further study the dependence of these results on the input parameters, we also analyzed the dependence on the initial angular velocity. In Table 2 we list the times at which the postmerger central star reaches the conditions suitable for a explosion to occur, for several magnetic field strengths (models A to D) and initial angular velocities. Clearly, the duration of this phase and the final outcome depend sensitively on the value of the adopted magnetic field, and to a lesser extent on the initial angular velocity.

\subsection{Accretion Rate Set by Viscous Timescale}

In the previous section we have assumed that the accretion rate on the white dwarf is given by the shorter timescale of the cooling mechanisms. In this section we present the results for the case in which the white dwarf accretes material at a rate set by the viscous timescale of the Keplerian disk.

For the sake of definiteness, in the following we discuss in detail, with the help of Figures 8 and 9, the time evolution of model $\mathrm{E}$, which is similar to model A, the only difference being that in this case the accretion rate is computed adopting the viscous timescale, while the rest of the initial conditions and assumptions are exactly the same. Furthermore, for this model we study two possibilities for the efficiency parameter $\xi_{\text {acc }}$ in Equation (3). The first of these possibilities corresponds to the case in which $\xi_{\text {acc }}=1.0$, that is, when matter is accreted on the remnant with the Keplerian velocity at the inner disk radius. The second one corresponds to the case in which matter is accreted on the remnant with the Keplerian velocity at the surface of the remnant, which we label as $\xi_{\mathrm{acc}}=\xi_{\mathrm{WD}}$ in the figures discussed below.

Shortly after the merger, due to the high accretion rates, $R_{\text {mag }}<R_{\text {WD }}$ (see the top panel of Figure 8 ). Hence, the only torque acting on the white dwarf is that resulting from accretion. For the set of parameters of model E, the initial accretion rate onto the white dwarf given by Equation (25) is large, even if an efficiency of $\varepsilon=0.1$ is adopted, $\dot{M}_{\mathrm{WD}} \sim$ $0.01 M_{\odot} \mathrm{s}^{-1}$. Consequently, the mass of the white dwarf rapidly increases during this phase. However, its central density only increases in the case in which matter is accreted with the Keplerian velocity of the remnant (blue line in Figure 9), whereas in the case in which $\xi_{\text {acc }}=1.0$ (green line in this figure) the central density of the white dwarf decreases.

The evolution of the central density of the remnant is the result of an intricate trade-off between the increase in mass, the change in the rotation period due to the acting torque, and the evolution of the moment of inertia of the remnant. The increase in mass of the remnant alone clearly would result in an increase of the central density. The change in the rotation speed due to the acting torque-which tends to increase the angular velocity-would result in a decrease of the central density, as the centrifugal force increases. Finally, since $I \propto M_{\mathrm{WD}} R_{\mathrm{WD}}^{2}$, as the mass of the white dwarf increases as a result of accretion, the moment of inertia would increase. However, since the mass-radius relationship for rotating white dwarfs depends crucially on the angular velocity, the radius of the remnant, hence $I$, ultimately depends on the acting torque. Furthermore, for super-Chandrasekhar white dwarfs the slope of the mass-radius relationship is very steep. Consequently, small changes in the mass can induce large variations of the radius of the remnant. The interplay between these factors is rather complex, but in general terms we find that the evolution of the moment of inertia is dominated by the variation of the radius of the remnant.

It is then clear that the crucial parameter that dictates the evolution of the central density of the remnant is $\xi_{\text {acc }}$. Specifically, when $\xi_{\text {acc }}=1.0$ is adopted, the remnant is spun up very rapidly by the acting torque (as is shown in the bottom panel of Figure 8), its radius increases notably (see the top panel of this figure), the moment of inertia increases markedly, and the central density first decreases. On the contrary, if matter is accreted onto the surface of the white dwarf with the same angular velocity of the remnant, the acting torque is 
Table 2

Carbon Ignition Location and Evolutionary Times of the Post-merger Remnants for Several Values of the Magnetic Field Strength and Initial Angular Velocity

\begin{tabular}{|c|c|c|c|c|c|c|c|c|c|}
\hline \multirow{2}{*}{$\begin{array}{l}\text { Model } \\
\Omega_{\mathrm{WD}}\left(\mathrm{s}^{-1}\right)\end{array}$} & \multirow{2}{*}{$B_{\mathrm{WD}}(\mathrm{G})$} & \multicolumn{7}{|c|}{$\Delta t(\mathrm{yr})$} & \multirow{2}{*}{ Carbon Ignition } \\
\hline & & 2.3 & 2.5 & 2.6 & 2.7 & 2.8 & 2.9 & 3.0 & \\
\hline$\overline{\mathrm{A}}$ & $10^{9}$ & $5.50 \times 10^{1}$ & $4.68 \times 10^{1}$ & $4.33 \times 10^{1}$ & $4.02 \times 10^{1}$ & $4.71 \times 10^{1}$ & $6.95 \times 10^{1}$ & $4.08 \times 10^{1}$ & Center \\
\hline $\mathrm{C}$ & $10^{7}$ & $2.81 \times 10^{4}$ & $3.95 \times 10^{4}$ & $2.09 \times 10^{4}$ & $1.66 \times 10^{4}$ & $1.34 \times 10^{4}$ & $1.59 \times 10^{4}$ & $1.50 \times 10^{4}$ & Center \\
\hline $\mathrm{D}$ & $10^{8}$ & $9.29 \times 10^{2}$ & $7.96 \times 10^{2}$ & $7.39 \times 10^{2}$ & $8.92 \times 10^{2}$ & $8.31 \times 10^{2}$ & $7.76 \times 10^{2}$ & $5.61 \times 10^{2}$ & Center \\
\hline
\end{tabular}
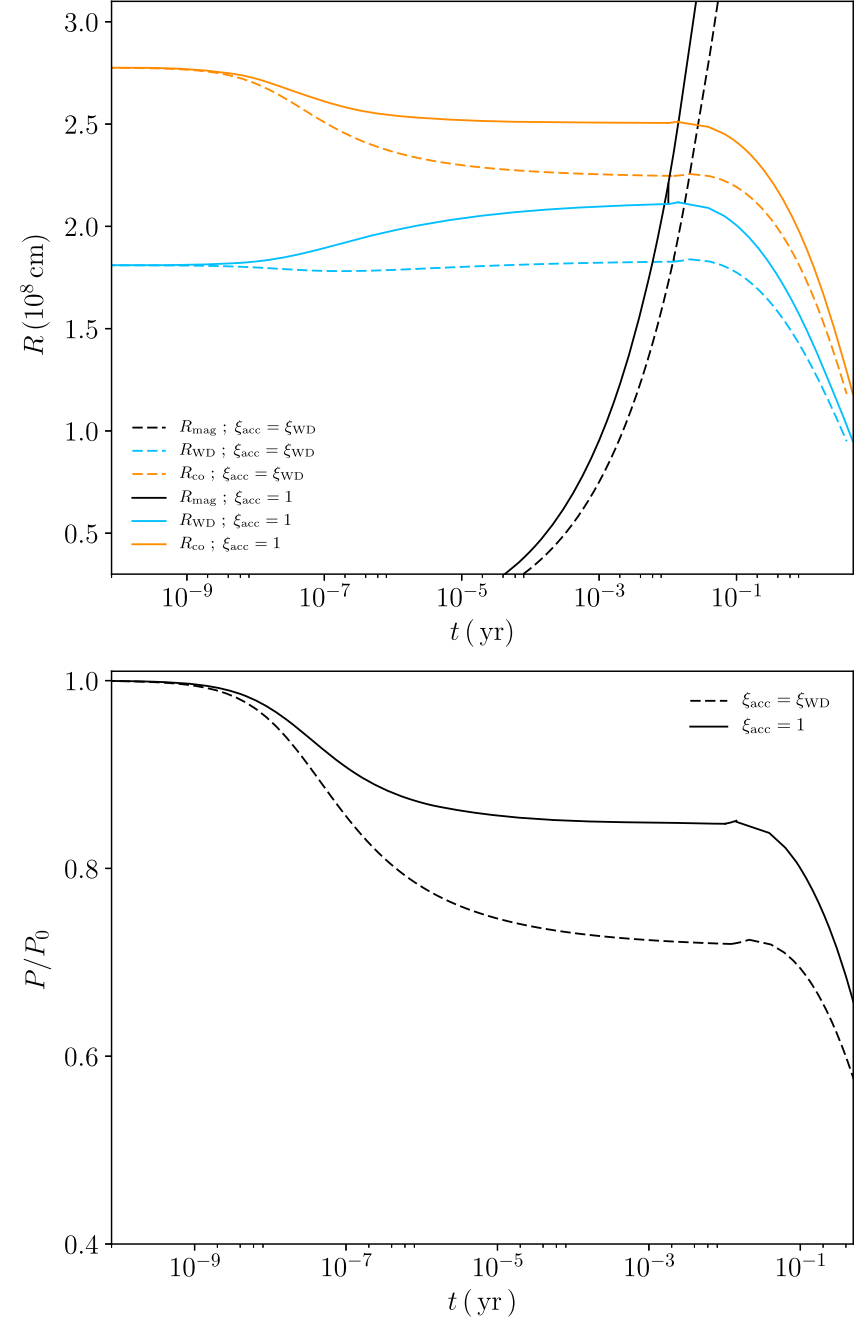

Figure 8. Top panel: evolution of the radius of the remnant $R_{\mathrm{WD}}$, the corotation radius $R_{\mathrm{co}}$, and the magnetospheric radius $R_{\mathrm{mag}}$ for model E. Bottom panel: evolution of the rotation period of the central white dwarf.

considerably smaller, the moment of inertia remains approximately constant, the radius of the remnant decreases, and the central density increases steadily. In both cases, once the magnetic radius becomes larger than the radius of the white dwarf $\left(R_{\mathrm{WD}}<R_{\mathrm{mag}}<R_{\mathrm{co}}\right)$, the dipole and the disk-interaction torques drive the evolution of the remnant-the top panel of Figure 8 clearly depicts this. When this happens, the fastness parameter of the white dwarf is $\sim 0.8$. Consequently, shortly after $(\lesssim 0.5 \mathrm{yr})$, the remnant reaches a fastness parameter $\omega_{\mathrm{f}}=1$, and the evolution is driven by the propeller torque. At this point of the evolution, accretion from the disk stops. Consequently, the moment of inertia of the remnant decreases considerably, the rotation period decreases as well, and the

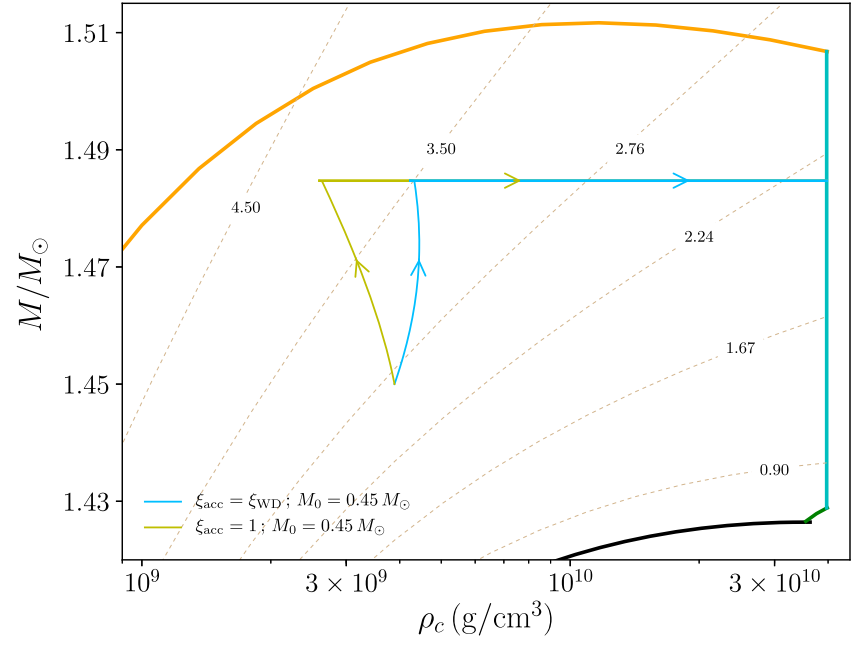

Figure 9. Evolutionary tracks in the mass-density plane for model E for two assumptions about the value of $\xi_{\text {acc }}$ and $\varepsilon=0.1$.

white dwarf contracts. Hence, the central density increases rapidly (see the top panel of Figure 8). This entire sequence of events ultimately leads the remnant to cross the line of inverse $\beta$-decay instability. It is interesting to note that the mass of the remnant when it crosses the instability line does not depend on the adopted valued of $\xi_{\text {acc }}$. This depends on the mass accretion rate onto the white dwarf, and consequently on the adopted efficiency parameter $\xi$. The parameter $\xi_{\text {acc }}$ is directly related to the change of angular momentum. Thus, $\xi_{\text {acc }}$ and $\xi$ play different roles in the evolution of the post-merger configuration, although its effect is combined as shown by Equation (3).

We now address our attention to thermal evolution of the post-merger remnant of model E. Figure 10 shows several temperature-density profiles at selected times after the merger took place. The top panel of this figure shows the case in which $\xi=\xi_{\text {acc }}$ is adopted, whereas the bottom panel corresponds to the case in which $\xi=1$ is employed. The initial temperature profiles are shown as the solid blue and green lines in Figure 10, respectively. In both cases, during the first evolutionary phases the large accretion rates discussed earlier heat the outer layers of the star. However, neutrino emission also plays a significant role. When $\xi=\xi_{\text {acc }}$ is adopted, compressional work exceeds neutrino emission, and thus an off-center temperature peak rapidly grows. In particular, the temperature profile peaks at $\log \rho \sim 6.54$. As time passes by, the peak temperature increases, and ultimately the outer layers of the white dwarf reach the thermodynamic conditions needed to ignite carbon explosively.

On the contrary, when $\xi=1.0$ is adopted (bottom panel of Figure 10), the evolution is more complex. In this case accretion first heats the outer layers of the central white dwarf, 

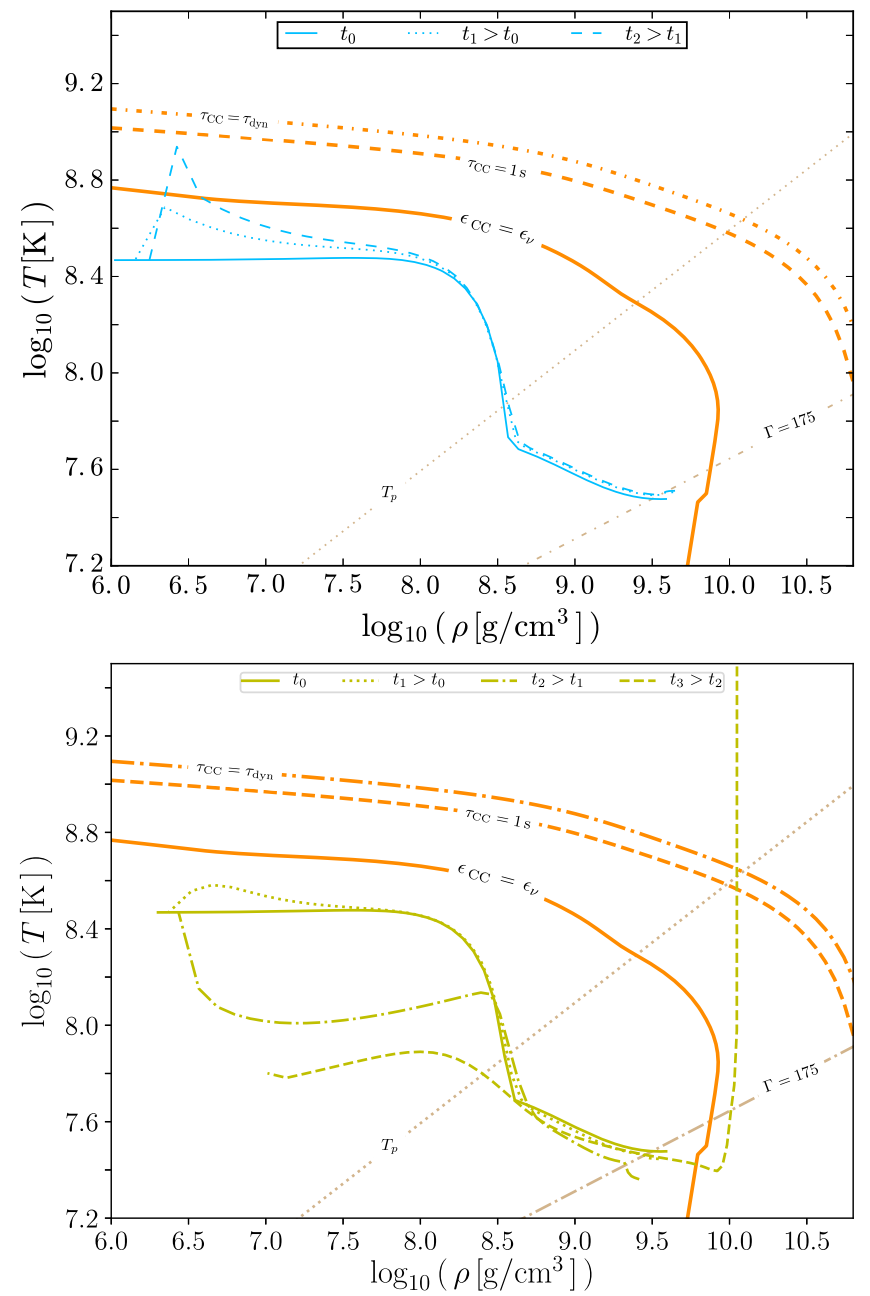

Figure 10. Temperature-density profiles of the central white dwarf at different times of the evolution for model $\mathrm{E}$. The top panel shows the model with $\xi=\xi_{\text {acc }}$, while the bottom panel displays the model for which $\xi=1$ is adopted. Time $t_{1}$ corresponds to a time shortly after accretion from the disk starts (short-dashed line), $t_{2}$ is the time at which the merged remnant enters into the propeller phase and accretion stops (dot-dashed line), and $t_{3}$ is a time just before the post-merger object crosses the beta-instability limit (longdashed line).

but neutrino cooling dominates. Hence, after a short time interval the entire central white dwarf cools to a temperature smaller than the initial one. Time $t_{2}$ is the time at which accretion stops. Thus, for times longer than $t_{2}$ the evolution of the star is driven by angular momentum losses by dipole emission. Eventually, at time $t_{3}$ carbon is ignited at the center of the white dwarf.

\subsubsection{Sensitivity of the Results to the Free Parameters}

We first compare the evolution when different masses of the disk are adopted, keeping the mass of the remnant fixed. With this in mind, in Figure 11 we show the evolutionary tracks of model $\mathrm{F}$ and compare them with those of model $\mathrm{E}$. This model corresponds to a final post-merger remnant of mass $1.45 M_{\odot}$ and an initial disk mass of $0.65 M_{\odot}$, whereas the rest of the parameters of the model were not varied-see Table 1 . Additionally, as we did for model E, we also studied two possibilities. The first of these corresponds to an accretion efficiency parameter $\xi_{\text {acc }}=1.0$, whereas for the second one we assumed that at the inner disk radius the accreted material has

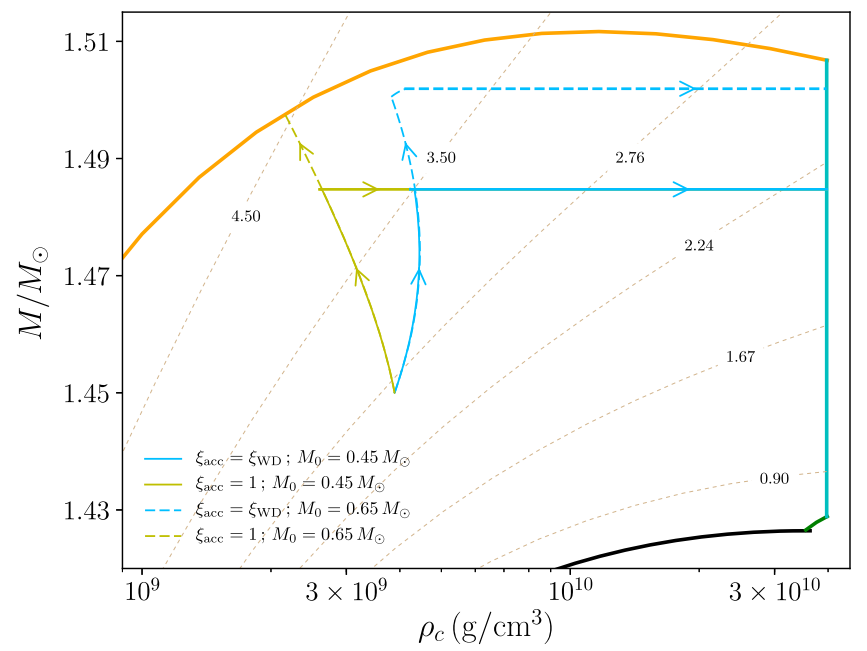

Figure 11. Same as Figure 9, but here we compare models $\mathrm{E}$ (solid lines) and $\mathrm{F}$ (dashed lines) for two different disk initial masses, 0.45 and $0.65 M_{\odot}$, respectively.

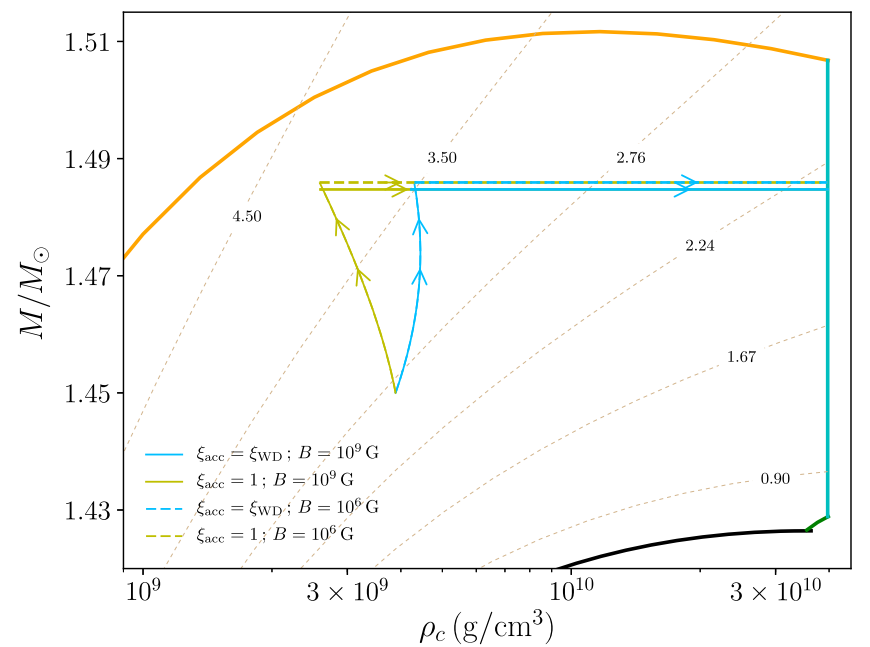

Figure 12. Same as Figure 9, but here we compare models E (solid lines) and $\mathrm{G}$ (dashed lines) for two different values of the white dwarf magnetic field, $10^{9}$ and $10^{6} \mathrm{G}$, respectively.

the angular velocity of the white dwarf, $\xi_{\mathrm{acc}}=\xi_{\mathrm{wD}}$. Note that, in the case in which $\xi_{\text {acc }}=0.1$ is adopted, the evolution of this model is very similar to that of model $\mathrm{E}$, but it arrives at the mass-shedding limit in the accretion phase. When $\xi_{\mathrm{acc}}=\xi_{\mathrm{wD}}$ is chosen, the remnant also crosses the line of inverse $\beta$-decay instability. The only difference is that for model $\mathrm{F}$, which has a disk considerably more massive, more mass is accreted before the remnant crosses the instability line. Hence, the remnant has a larger mass in the propeller phase, and the line of $\beta$-decay instability is crossed when the white dwarf is more massive.

Next, we analyze the influence on the evolution of the strength of the magnetic field, as we did previously for those models in which the accretion rate is computed using the cooling timescale. We do this comparison adopting a very low value for the magnetic field strength, $B=10^{6} \mathrm{G}$ (model $\mathrm{G}$ in Table 1, the same value adopted in Section 5.1). The results of this analysis are shown in Figure 12. As can be seen, the differences between the evolution of models $E$ and $G$ are minor. Thus, we conclude that for the typical values of the magnetic field strength that originated in the merger of two white dwarfs, the evolution of these models is not significantly 


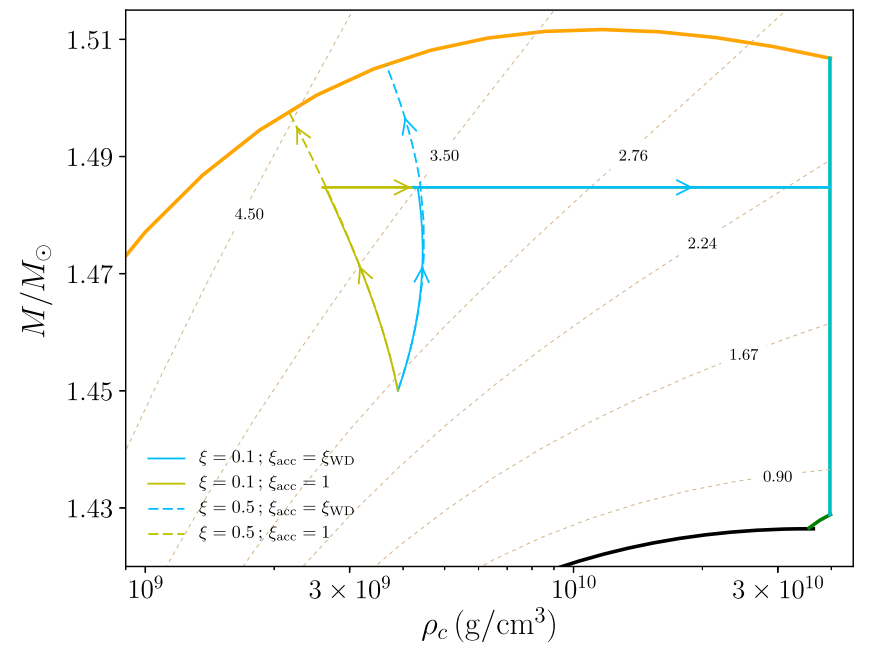

Figure 13. Same as Figure 9, but for two values of the accretion efficiency parameter, $\varepsilon=0.1$ (solid lines) and 0.5 (dashed lines), for models $\mathrm{E}$ and $\mathrm{H}$, respectively.

affected by the adopted magnetic field. This can be explained by the fact that, at early times in the viscous timescale prescription, the mass and angular momentum of the white dwarf post-merger evolution are dominated by the accretion torque and the effect of the magnetic field is neglected until the magnetospheric radius equals the white dwarf one. It is in this initial phase that more significant accretion of mass onto the central star occurs. Since the accretion timescale in this scenario is so short, the evolution of the magnetospheric radius is dominated by the change of the mass accretion rate.

Another free parameter of our formulation is $\varepsilon$, which, we recall, measures how efficient accretion is. All the calculations presented until now have been performed adopting $\varepsilon=0.1$. However, since the accreted mass depends significantly on the precise value of this free parameter, it is important to assess its impact on the results. We now study the sensitivity of our calculations to the value adopted for it. To this end we conducted an additional set of simulations in which we adopted $\varepsilon=0.5$. This is model $\mathrm{H}$ in Table 1 . In Figure 13 we compare the results of these calculations with those of model E. As could be expected, this parameter turns out to be critical, since it controls how efficient accretion is. Consequently, when $\varepsilon=0.5$ is adopted, the remnant evolves toward the massshedding limit instead of crossing the $\beta$-instability line. We consider model $\mathrm{E}$ as a reasonable guess, although keeping in mind that larger values of $\varepsilon$ cannot be discarded "a priori" and therefore could alter the evolution of the remnant.

Naturally, another key ingredient of our approach is the initial angular velocity of the central white dwarf, $\Omega_{\mathrm{WD}}$. Figure 14 shows the evolutionary tracks for different values of the initial angular velocity of the post-merger remnant, keeping unchanged the rest of initial conditions of model E (models I to $\mathrm{Q}$ in Table 1; dashed lines), and we compare them with the evolutionary sequence of model $\mathrm{E}$, our reference model for this prescription for the accretion rate (solid lines). As can be seen, the model with the smallest initial angular velocity-namely, that with initial angular velocity $2.30 \mathrm{rad} \mathrm{s}^{-1}$, model I-reaches the mass-shedding limit during the accretion phase. Furthermore, for this model the central density decreases, irrespective of the value adopted for $\xi_{\text {acc }}$. Model $\mathrm{J}$, for which we adopted $\Omega_{\mathrm{WD}}=2.50 \mathrm{rad} \mathrm{s}^{-1}$, only reaches the mass-shedding limit if $\xi_{\text {acc }}=1.0$; otherwise, it reaches the $\beta$ instability region. The

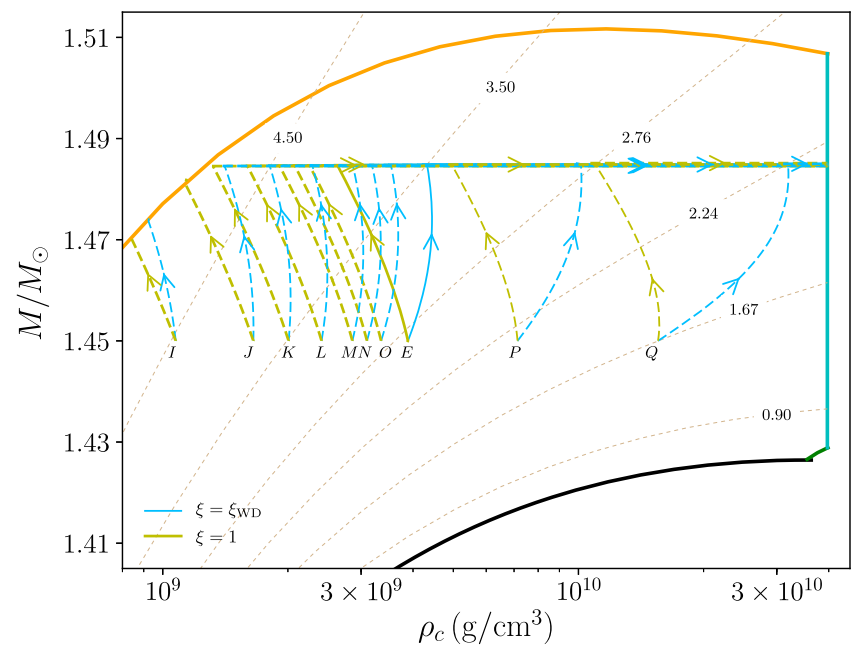

Figure 14. Same as Figure 9, but for several values of the initial angular velocity, models I to $\mathrm{P}$ in Table 1, compared to our reference case, for which the initial angular velocity is $\Omega_{\mathrm{WD}}=3.00$ (model E). From left to right the initial angular velocities of the remnant are $\Omega_{\mathrm{WD}}=2.30,2.50,2.60,2.70,2.80$, $2.85,2.90,3.50$, and $4.50 \mathrm{~s}^{-1}$.

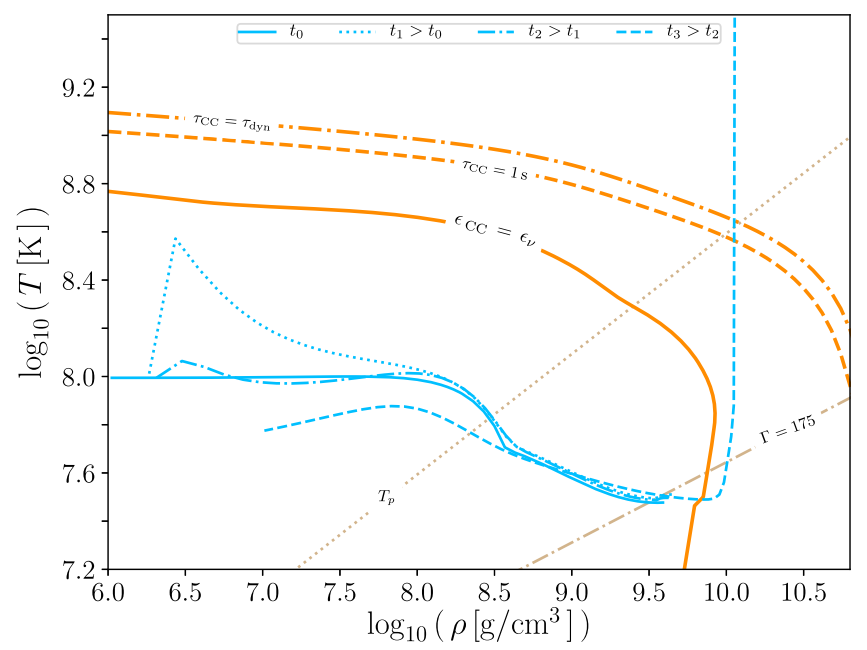

Figure 15. Same as Figure 10, but for the case in which the initial temperature of model E is smaller. For the sake of conciseness we only display the model for which $\xi=1$ is adopted.

rest of the models do not cross the mass-shedding instability line. Note as well that for model $\mathrm{J}$, as occurs for model I, the central density decreases, independently of the value adopted for $\xi_{\text {acc }}$. For model $\mathrm{K}$ the central density decreases if $\xi_{\mathrm{acc}}=1.0$ and increases otherwise. This is also true for models with even faster rotation rates.

Additionally, to take into account the effects of the initial temperature for the case in which $\xi=\xi_{\text {acc }}$ is adopted, we computed a model in which the initial configuration corresponds to a white dwarf in which the external layers have a temperature sizably smaller (i.e., $10^{8} \mathrm{~K}$ ) than that of our standard case-that is, model E-which was computed adopting a temperature for the external layers of $3 \times 10^{8} \mathrm{~K}$. The evolution of this additional model is shown in Figure 15, and it is markedly different from that of model E. Specifically, when the adopted temperature is $10^{8} \mathrm{~K}$, the external layers of the white dwarf are initially heated by the accreted material, as happens also for model E. However, in this case neutrino cooling (basically dominated by neutrino bremsstrahlung) is so 

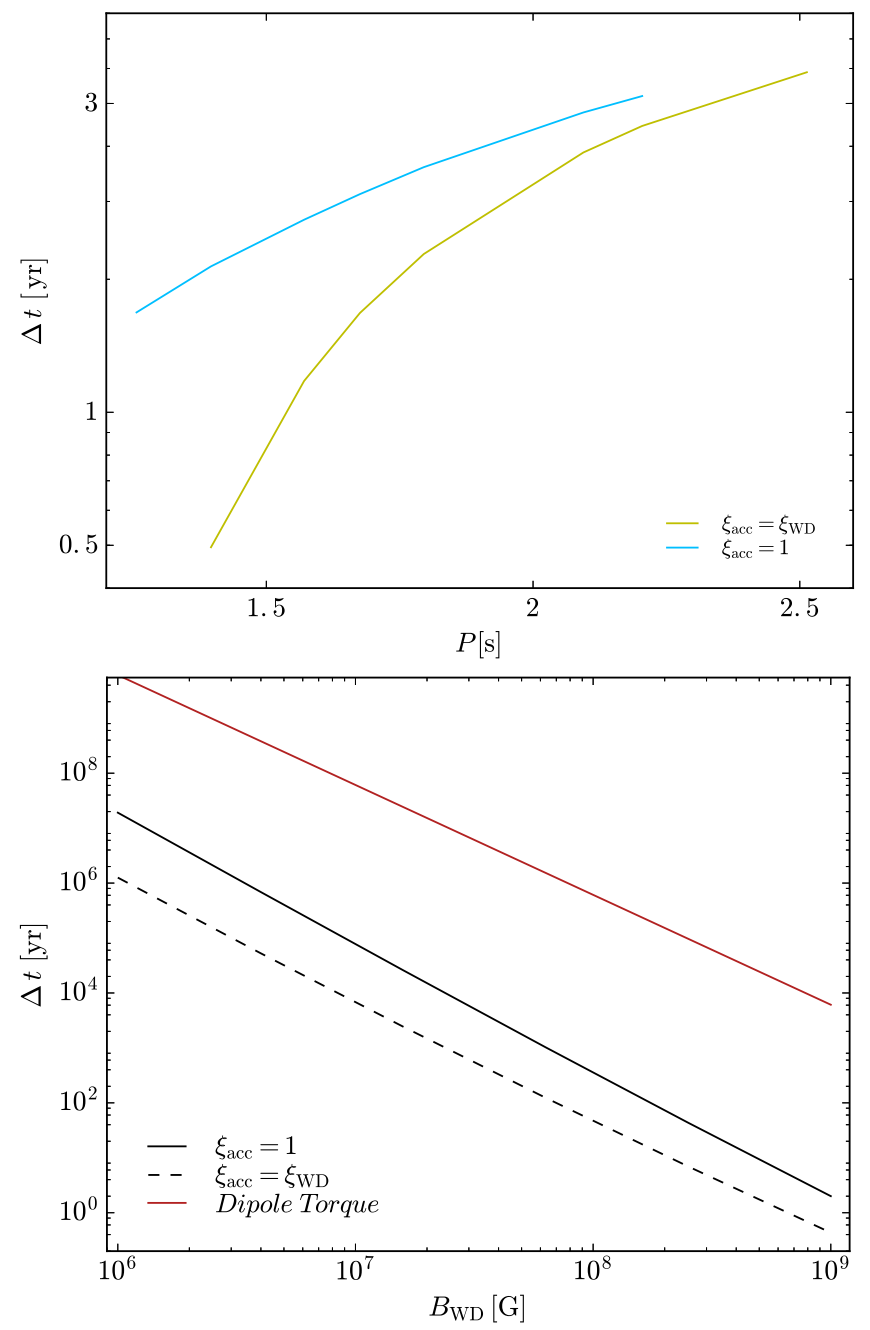

Figure 16. Top panel: time necessary to reach the instability line as a function of the initial period of the remnant. Bottom panel: time necessary to reach the instability line as a function of the magnetic field of the remnant.

strong that the remnant cools rapidly and eventually carbon is ignited at the center of the star, when the central regions have already crystallized.

\subsubsection{Evolutionary Times}

Finally, we study the time needed to reach the instability lines, $\Delta t$. We have shown before that the most important parameter that determines the evolution of the system is the initial period of the system. In the top panel of Figure 16 the dependence of $\Delta t$ on the initial period of the rotating white dwarf resulting from the merger is displayed, for our two choices for the value of $\xi_{\text {acc }}$. Clearly, the larger the period is, the longer $\Delta t$ is. This is the natural consequence of the smaller initial centrifugal force.

The second important parameter that determines the duration of this evolutionary phase is the strength of the magnetic field of the remnant. We showed before that the value of $B_{\mathrm{WD}}$ has little effect on the outcome of the post-merger system-see Figure 12. Notwithstanding, $B_{\mathrm{WD}}$ influences considerably $\Delta t$. This occurs because the larger $B_{\mathrm{WD}}$ is, the larger is the magnetospheric radius. Thus, we expect that large values of $B_{\mathrm{WD}}$ would result in substantially smaller values of $\Delta t$. To illustrate this in a quantitative manner, in the bottom panel of
Figure 16 the total time needed to reach the instability region is plotted as a function of the surface magnetic field of the remnant. As can be seen, the evolution of the systems is indeed faster for large magnetic field strengths. This behavior is natural since for large magnetic field strengths the central remnant is more tightly coupled with the surrounding Keplerian disk. Consequently, the accretion phase is shorter. In addition, in this panel we also show (using a solid red line) the time it takes for the remnant to become unstable when only magnetic dipole braking is considered. Since, in this case, there is no accretion, the star evolves in a constant-mass sequence. From Equation (7), we obtained

$$
\Delta t=\frac{c^{3}}{2 B_{\mathrm{WD}}^{2}} \int_{J_{\mathrm{WD}, 0}}^{J_{\mathrm{WD}, f}} \frac{d J_{\mathrm{WD}}}{\Omega_{\mathrm{WD}}^{3} R_{\mathrm{WD}}^{6}} .
$$

It can be seen that $\Delta t$ is inversely proportional to the square of the magnetic field. Ilkov \& Soker (2012) give an analytical expression of Equation (30) in the approximate case when changes in the white dwarf structure due to the configuration rearrangement during the angular momentum loss are neglected. It is important to mention that such changes are very important for the determination of the rotational evolution of super-Chandrasekhar white dwarfs (Boshkayev et al. 2013). Clearly, the evolution of the system is always much faster when all the torques acting on the remnant are correctly taken into account. Furthermore, it is important to realize that the evolution is even faster when matter is accreted onto the surface of the remnant with the Keplerian velocity. Finally, note as well that when $\xi_{\text {acc }}=1.0$ is adopted, moderately longer durations of the post-merger phase, when compared with the case in which the accreted matter has the Keplerian velocity at the surface of the remnant, are obtained.

\section{Comparison with Previous Works}

A detailed study of the evolution of the remnant of white dwarf mergers started with the work of Yoon et al. (2007). They mapped the final configuration of the SPH simulations of the merger of a $0.9-0.6 M_{\odot}$ binary into a $1 \mathrm{D}$ stellar evolution hydrodynamic code and followed its forward evolution. Their merger remnant is represented by a cold, slowly rotating rigid core surrounded by a hot, rapid rotating envelope with a Keplerian disk around it. They allowed the accretion from the disk by a constant accretion rate (of the order of the Eddington limit) and included a prescription for the transport of angular momentum with timescales of the order of the thermal timescale. They conclude that an off-center carbon ignition can be avoided if the transport angular momentum timescale is greater than the cooling timescale by neutrino emission and the accretion rate on the star is slow enough, i.e., $\dot{M}_{\text {WD }}<5 \times 10^{-6}-10^{-5} M_{\odot} \mathrm{yr}^{-1}$. We improved this approach by allowing the mass accretion rate to vary with time, consistently with the white dwarf thermal evolution. Additionally, we introduced a framework for evolution of the angular momentum of the post-merger white dwarf including the torque that acts on the star, taking into account the magnetic field effect of the central white dwarf on the evolution of the postmerger configuration. We have found that strong magnetic fields, $B_{\mathrm{WD}}>10^{7} \mathrm{G}$, can also avoid an off-center carbon ignition. 
Van Kerkwijk et al. (2010) estimated the post-merger evolution based on the results of the SPH simulations of Lorén-Aguilar et al. (2009) and concluded that the accretion occurs in a shorter timescale $(\sim 10 \mathrm{hr})$, causing a compression of the white dwarf core, with a consequent increase of its temperature until it reaches the carbon ignition runaway, leading to a delayed explosion. However, they focus on masssymmetric white dwarf mergers $\left(0.6-0.6 M_{\odot}\right)$, in which the two white dwarfs are disrupting and the final remnant configuration has a temperature profile that peaks at the center.

A different approach was presented in Shen et al. (2012) and Schwab et al. (2012, 2016). In these works it was argued that, due to the differential rotation of the system, after the dynamical phase of the merger the magnetorotational instability becomes effective, and that in a viscous timescale of $10^{4} \mathrm{~s}$ (orders of magnitude shorter than the thermal timescale of $\sim 10^{4} \mathrm{yr}$ ) the angular momentum of the tidally disrupted white dwarf can be redistributed in the surface of the central star, leading to a rotating configuration with a hot envelope and with almost all the mass of the secondary star. These works also computed the thermal evolution of the post-merger remnant characterized by a timescale of the order of $10^{3}-10^{4} \mathrm{yr}$. Schwab et al. (2012) studied the merger of a $0.9-0.6 M_{\odot}$ white dwarf binary, and Schwab et al. $(2012,2016)$ extended the initial conditions' parameter space, but the evolution of the postmerger configuration in the presence of a magnetic field of the central white dwarf was not considered. These configurations spin down during the viscous evolution, while our superChandrasekhar remnants spin up during the entire evolution, even in the case of angular momentum losses.

There are two works considering the effect of the magnetic field during the dynamical timescale of the merger (Zhu et al. 2015) and in the viscous timescale of the post-merger evolution ( $\mathrm{Ji}$ et al. 2013). In these works, the evolution of the system magnetic field is followed in a different fashion with respect to our approach, since we have adopted a dipole magnetic field with constant magnitude and inclination angle. Assuming as an initial condition the remnant of the merger of a 0.6-0.6 $M_{\odot}$ carbon-oxygen white dwarf binary from the SPH simulation of Lorén-Aguilar et al. (2009), Ji et al. (2013) evolve the system for $3 \times 10^{4} \mathrm{~s}$ with the FLASH code in a 2D axisymmetric cylindrical Eulerian grid. They introduced a weak poloidal magnetic field and showed that the magnetorotational instability developed in the disk leads to a rapid growth of its magnetic field, the spin-down of the white dwarf remnant, and its magnetization to field strengths around $\sim 2 \times 10^{8}$ G. They computed an effective magnetic ShakuraSunyaev parameter of the order of $\left\langle\alpha_{m}\right\rangle \sim 0.01$, a value one order of magnitude smaller than the $\alpha$ we adopted here. They found that the white dwarf magnetic field varies with time, indicating a disordered interior magnetic field. At the end of the simulation the magnetic field toroidal component is 1.5 times bigger than the poloidal one. In our model we have assumed a dipole magnetic field aligned with the white dwarf rotation axis. In this case, the magnetic field has no toroidal component. However, the simulations of $\mathrm{Ji}$ et al. (2013) have limited resolution, and the field strengths are affected by numerical resistivity. In addition, in these magnetohydrodynamics simulations the disk lost almost $90 \%$ of its initial mass, with $82 \%$ of it accreted by the white dwarf remnant and the rest going into outflows of which about $10^{-3} M_{\odot}$ are ejected and unbound to the system. The central white dwarf spins down, losing angular momentum owing to the development of Maxwell stresses at the white dwarf boundary. A direct comparison is difficult, since we have studied super-Chandrasekhar white dwarfs with initial angular velocity one order of magnitude higher than that studied by Ji et al. (2013) $\left(\Omega_{\mathrm{WD}, 0}=0.03 \mathrm{~s}^{-1}\right)$. However, one difference is that in our model the white dwarf first gains angular momentum owing to the mass accretion and then loses it owing to the magnetic torque.

Zhu et al. (2015) simulated the merger of a $0.625-0.65 M_{\odot}$ carbon-oxygen white dwarf binary, giving to each white dwarf a dipole seed magnetic field with the moving-mesh code AREPO (Springel 2010). They found that during the merger dynamics the magnetic field was too weak to have an effect on the evolution, obtaining a final remnant composed by a degenerate core with a thermally supported envelope surrounded by a rotationally supported disk. This configuration is similar to the one obtained in the SPH simulations (Zhu et al. 2013). However, the remnant magnetic field has a complex structure with a volume-averaged field strength $>10^{10} \mathrm{G}$ in the core, with both poloidal and toroidal components.

Table 3 summarizes the main features of the post-merger evolution studied in this paper and the comparison with those of the works discussed above. We specified the binary mass of the merger configurations studied, the post-merger configurations after the merger (for the works of Yoon et al. 2007; van Kerkwijk et al. 2010; Zhu et al. 2013) or after the viscous evolutions of the system (for the works of Schwab et al. 2012; Shen et al. 2012; Ji et al. 2013), and the magnitude and configuration of the central remnant magnetic field (if it is considered). In the last column, we specified whether the central remnant developed conditions for an off-center or center carbon ignition.

\section{Conclusions}

The evolution of the remnant of the merger of a binary white dwarf is still an open problem. Detailed hydrodynamical simulations show that the product of the merger consists of a central white dwarf that rotates as a rigid body, surrounded by a hot, rapidly rotating corona-which has been proven to produce large magnetic fields - and a Keplerian disk. In this paper we studied the evolution of metastable, magnetized super-Chandrasekhar white dwarfs formed in the aftermath of the merger of close binary systems made of two white dwarfs.

Our simulations take into account the magnetic torques acting on the star, accretion from the Keplerian disk, and the threading of the magnetic field lines through the disktherefore improving previous calculations of this kind. Furthermore, in our computations - at odds with previous efforts-we employ a mass-radius relationship for rotating white dwarfs. Also, our calculations incorporate the thermal evolution of the white dwarf.

Furthermore, our simulations were performed using two different prescriptions for the mass accretion rate on the central white dwarf. In a first set of simulations we adopted an accretion rate set by the cooling timescale of the Keplerian disk, whereas in the second suite of models the adopted accretion rate was computed using the viscous timescale of the disk. These two prescriptions for the accretion rate cover a very large range of values and therefore allow us to investigate the 
Table 3

Comparison with Previous Works

\begin{tabular}{|c|c|c|c|c|c|}
\hline Work & Merger Configuration & Post-merger Configurations & $\begin{array}{l}\text { Magnetic } \\
\text { Field }\end{array}$ & $\begin{array}{l}\text { Magnetic Field } \\
\text { Configuration }\end{array}$ & Carbon Ignition \\
\hline Yoon et al. (2007) & $0.9 M_{\odot}-0.6 M_{\odot}, 0.9 M_{\odot}-0.7 M_{\odot}, 1.0 M_{\odot}-1.0 M_{\odot}$ & $\begin{array}{l}\text { Slowly rotating cold core }\left(0.6 M_{\odot}\right) \text { with a rapidly } \\
\text { rotating hot envelope }\left(0.5 M_{\odot}\right)+\text { a Keplerian disk } \\
\left(0.4 M_{\odot}\right)\end{array}$ & $\cdots$ & $\cdots$ & Center/off-center ignition \\
\hline $\begin{array}{l}\text { van Kerkwijk } \\
\text { et al. (2010) }\end{array}$ & $0.6 M_{\odot}-0.6 M_{\odot}, \mathrm{C} / \mathrm{O} \mathrm{WD}$ & Rapidly rotating WD + thick disk & $\cdots$ & $\ldots$ & Center ignition \\
\hline Shen et al. (2012) & $0.6 M_{\odot}-0.9 M_{\odot}, \mathrm{C} / \mathrm{O} \mathrm{WD}$ & $\begin{array}{l}\text { WD }\left(\sim 0.9 M_{\odot}\right) \text { with a hot, slowly rotating and radially } \\
\text { extended envelope supported by thermal pressure } \\
\left(\sim 0.6 M_{\odot}\right)\end{array}$ & $\cdots$ & $\cdots$ & $\begin{array}{l}\text { Off-center ignition that leads } \\
\text { to a high-mass } \mathrm{O} / \mathrm{Ne} \mathrm{WD}\end{array}$ \\
\hline $\begin{array}{l}\text { Schwab et al. (2012)/ } \\
\text { Schwab et al. (2016) }\end{array}$ & $0.6 M_{\odot}-0.9 M_{\odot}, 0.9 M_{\odot}-1.2 M_{\odot}, \mathrm{C} / \mathrm{O} \mathrm{WD}$ & WD with a thermally supported envelope $\left(1.5 M_{\odot}\right)$ & $\cdots$ & $\cdots$ & $\begin{array}{l}\text { Off-center ignition that leads } \\
\text { to an } \mathrm{O} / \mathrm{Ne} \mathrm{WD}\end{array}$ \\
\hline Ji et al. (2013) & $0.6 M_{\odot}-0.6 M_{\odot}, \mathrm{C} / \mathrm{O} \mathrm{WD}$ & $\begin{array}{l}\text { Rotating WD }\left(0.96 M_{\odot}\right) \text { surrounded by a hot corona } \\
\quad\left(0.04 M_{\odot}\right)+\text { thick disk }\left(0.2 M_{\odot}\right)\end{array}$ & $2 \times 10^{8} \mathrm{G}$ & $\frac{B_{t}}{B_{p}} \sim 1.5$ & Center ignition \\
\hline Zhu et al. (2015) & $0.625 M_{\odot}-0.65 M_{\odot}, \mathrm{C} / \mathrm{O} \mathrm{WD}$ & $\begin{array}{l}\text { WD }\left(0.64 M_{\odot}\right) \text { surrounded by thermally supported hot } \\
\text { envelope }\left(0.42 M_{\odot}\right)+\operatorname{disk}\left(0.21 M_{\odot}\right)\end{array}$ & $4 \times 10^{10} \mathrm{G}$ & $\frac{E_{B t}}{E_{B}}=0.6$ & $\ldots$ \\
\hline This work & $0.78 M_{\odot}-1.12 M_{\odot}, \mathrm{C} / \mathrm{O} \mathrm{WD}$ & $\begin{array}{l}1.45 M_{\odot} \text { rigidly rotating super-Chandrasekhar WD + } \\
0.45 M_{\odot} \text { thin Keplerian disk }\end{array}$ & {$\left[10^{6}, 10^{9}\right] \mathrm{G}$} & Poloidal & Center/off-center ignition \\
\hline
\end{tabular}

Note. $B_{t} / B_{p}$ : ratio of the toroidal to the poloidal field strength. $E_{B t} / E_{B}$ : ratio of the toroidal to the total magnetic energy. 
possible outcomes of the evolution of these systems in a quite generic way.

Finally, we also explored the effects of the adopted set of initial parameters. These include the mass of the remnant star, its radius, angular velocity, and moment of inertia, as well as the strength of the magnetic field. We showed that the timescale in which the newly formed white dwarf evolves to reach the thermodynamical conditions for carbon to be burned explosively, or to reach the Keplerian mass shedding, secular axisymmetric instability, or inverse beta-decay instability depends crucially on all these parameters.

However, we have made a number of assumptions that might be relaxed in forthcoming works. For instance, we allow a nonzero inclination angle between the spin axis and the orientation of the dipole magnetic field and its evolution with time. Also, the post-merger central remnant rotates differentially between the core and the corona, while we have adopted a totally rigid central remnant. Thus, the model can be improved by implementing a transport mechanism for the angular momentum in the interior of the central white dwarf remnant.

For the accretion rate prescription set by the viscous timescale, we have adopted a geometrically thin disk model. At the early times in the evolution of the post-merger remnant, we obtain a highly super-Eddington accretion rate of up to $10^{-1} M_{\odot} \mathrm{s}^{-1}$. The dissipation required to produce the needed very short viscous timescales might heat the disk to the point of carbon ignition (Mochkovitch \& Livio 1990), but we did not consider these heating effects in the present work. The disk of the post-merger remnant could be best described by a thickdisk model. This might also be studied in a forthcoming work.

On the other hand, we have approximated the white dwarf magnetic field to a prefect dipole configuration, but this is not necessarily the case, as has been shown by the MHD simulations of Ji et al. (2013) and Zhu et al. (2015). This work could be extended in order to introduce additional configurations of the magnetic field and its interaction with the surrounding disk.

We showed that in most of our models carbon reactions are highly efficient in heating the interior of the remnant, with timescales shorter than the dynamical time. This can happen either in the outer layers or at the center of the newly formed white dwarf, depending on the initial conditions of the white dwarf and on the efficiency of mass accretion and angular momentum evolution (see Figure 10). In most cases, the time it takes the star to reach explosive conditions is shorter than that needed to reach the inverse beta-decay instability or the secular instability, which lead to gravitational collapse. Hence, we conclude that the central white dwarf can reach conditions for a delayed explosion for a sufficiently broad set of initial conditions. Whether carbon is ignited at the center or is burned off-center depends crucially on the magnetic field strength and is almost independent of the post-merger WD initial angular velocity. Our simulations show that when the magnetic field is weak, carbon is ignited off-center, while central explosions are the outcome when an ordered global strong dipole magnetic field is produced in the hot, rapidly rotating convective corona formed in the aftermath of white dwarf mergers. Naturally, this depends on whether the stellar dynamo is saturated or not.

In summary, spinning, magnetized, super-Chandrasekhar white dwarfs, resulting from the merger of two less massive white dwarfs that do not produce an SN Ia in a violent merger, can produce a delayed explosion, provided that the remnant is massive enough and a strong magnetic field is produced during the merger.

During the refereeing process of this work our colleague and dear friend Enrique García-Berro sadly passed away. To him and to his memory, we dedicate this work and express all our gratitude. We thank the referee for the detailed comments and suggestions, which helped improve the presentation of our results. Part of this work was supported by MINECO grant AYA2014-59084-P and by the AGAUR.

\section{ORCID iDs}

J. A. Rueda (i) https://orcid.org/0000-0003-4904-0014

P. Lorén-Aguilar (iD https://orcid.org/0000-0001-8627-6478

E. García-Berro (i) https://orcid.org/0000-0002-1623-5838

\section{References}

Aznar-Siguán, G., García-Berro, E., Lorén-Aguilar, P., José, J., \& Isern, J. 2013, MNRAS, 434, 2539

Aznar-Siguán, G., García-Berro, E., Lorén-Aguilar, P., Soker, N., \& Kashi, A. 2015, MNRAS, 450, 2948

Beloborodov, A. M. 2014, MNRAS, 438, 169

Benz, W., Cameron, A. G. W., Press, W. H., \& Bowers, R. L. 1990, ApJ, 348, 647

Boshkayev, K., Rueda, J. A., Ruffini, R., \& Siutsou, I. 2013, ApJ, 762, 117

Cannizzo, J. K., Lee, H. M., \& Goodman, J. 1990, ApJ, 351, 38

Chabrier, G., \& Potekhin, A. Y. 1998, PhRvE, 58, 4941

Chandrasekhar, S. 1931, ApJ, 74, 81

Chandrasekhar, S. 1970, ApJ, 161, 571

Chatterjee, P., Hernquist, L., \& Narayan, R. 2000, ApJ, 534, 373

Dan, M., Rosswog, S., Brüggen, M., \& Podsiadlowski, P. 2014, MNRAS, 438, 14

Dan, M., Rosswog, S., Guillochon, J., \& Ramirez-Ruiz, E. 2011, ApJ, 737, 89

Davidson, K., \& Ostriker, J. P. 1973, ApJ, 179, 585

Eggleton, P. P. 1983, ApJ, 268, 368

Ertan, Ü., Ekşi, K. Y., Erkut, M. H., \& Alpar, M. A. 2009, ApJ, 702, 1309

Friedman, J. L., Ipser, J. R., \& Sorkin, R. D. 1988, ApJ, 325, 722

García-Berro, E., Lorén-Aguilar, P., Aznar-Siguán, G., et al. 2012, ApJ, 749,25

Gasques, L. R., Afanasjev, A. V., Aguilera, E. F., et al. 2005, PhRvC, 72, 025806

Ghosh, P., \& Lamb, F. K. 1979, ApJ, 232, 259

Goldreich, P., \& Julian, W. H. 1969, ApJ, 157, 869

Guerrero, J., García-Berro, E., \& Isern, J. 2004, A\&A, 413, 257

Han, Z., \& Podsiadlowski, P. 2004, MNRAS, 350, 1301

Hartle, J. B. 1967, ApJ, 150, 1005

Hartle, J. B., \& Thorne, K. S. 1968, ApJ, 153, 807

Henyey, L., \& L'Ecuyer, J. 1969, ApJ, 156, 549

Iben, I., Jr., \& Tutukov, A. V. 1984, ApJS, 54, 335

Ilkov, M., \& Soker, N. 2012, MNRAS, 419, 1695

Ilkov, M., \& Soker, N. 2013, MNRAS, 428, 579

Illarionov, A. F., \& Sunyaev, R. A. 1975, A\&A, 39, 185

Itoh, N., Hayashi, H., Nishikawa, A., \& Kohyama, Y. 1996, ApJS, 102, 411

Itoh, N., Kohyama, Y., Matsumoto, N., \& Seki, M. 1984, ApJ, 285, 758

Itoh, N., Mitake, S., Iyetomi, H., \& Ichimaru, S. 1983, ApJ, 273, 774

Ji, S., Fisher, R. T., García-Berro, E., et al. 2013, ApJ, 773, 136

Kashi, A., \& Soker, N. 2011, MNRAS, 417, 1466

Külebi, B., Ekşi, K. Y., Lorén-Aguilar, P., Isern, J., \& García-Berro, E. 2013, MNRAS, 431, 2778

Leonard, D. C. 2007, ApJ, 670, 1275

Livio, M., \& Riess, A. G. 2003, ApJL, 594, L93

Lorén-Aguilar, P., Isern, J., \& García-Berro, E. 2009, A\&A, 500, 1193

Menou, K., Esin, A. A., Narayan, R., et al. 1999, ApJ, 520, 276

Mochkovitch, R., \& Livio, M. 1990, A\&A, 236, 378

Nomoto, K. 1982, ApJ, 253, 798

Nomoto, K., \& Iben, I., Jr. 1985, ApJ, 297, 531

Pakmor, R., Kromer, M., Röpke, F. K., et al. 2010, Natur, 463, 61

Perlmutter, S., Aldering, G., Goldhaber, G., et al. 1999, ApJ, 517, 565

Phillips, M. M. 1993, ApJL, 413, L105

Piersanti, L., Gagliardi, S., Iben, I., Jr., \& Tornambé, A. 2003, ApJ, 583, 885 
Potekhin, A. Y., \& Chabrier, G. 2000, PhRvE, 62, 8554

Pringle, J. E. 1974, PhD thesis, Univ. Cambridge

Raskin, C., Scannapieco, E., Fryer, C., Rockefeller, G., \& Timmes, F. X. 2012 ApJ, 746, 62

Riess, A. G., Filippenko, A. V., Challis, P., et al. 1998, AJ, 116, 1009

Rotondo, M., Rueda, J. A., Ruffini, R., \& Xue, S.-S. 2011, PhRvD, 84, 084007

Saio, H., \& Nomoto, K. 1985, A\&A, 150, L21

Salpeter, E. E. 1961, ApJ, 134, 669

Sato, Y., Nakasato, N., Tanikawa, A., et al. 2015, ApJ, 807, 105

Schwab, J., Quataert, E., \& Kasen, D. 2016, MNRAS, 463, 3461

Schwab, J., Shen, K. J., Quataert, E., Dan, M., \& Rosswog, S. 2012, MNRAS, 427, 190

Shakura, N. I., \& Sunyaev, R. A. 1973, A\&A, 24, 337

Shen, K. J., Bildsten, L., Kasen, D., \& Quataert, E. 2012, ApJ, 748, 35

Shibata, M., Baumgarte, T. W., \& Shapiro, S. L. 2000, PhRvD, 61, 044012
Spitkovsky, A. 2006, ApJL, 648, L51

Springel, V. 2010, MNRAS, 401, 791

Stergioulas, N. 2003, LRR, 6, 3

Totani, T., Morokuma, T., Oda, T., Doi, M., \& Yasuda, N. 2008, PASJ, 60, 1327

Townsley, D. M., \& Bildsten, L. 2004, ApJ, 600, 390

van Kerkwijk, M. H., Chang, P., \& Justham, S. 2010, ApJL, 722, L157

Wang, Y.-M. 1987, A\&A, 183, 257

Wang, Y.-M. 1995, ApJL, 449, L153

Wang, Y.-M. 1997, ApJL, 475, L135

Webbink, R. F. 1984, ApJ, 277, 355

Weinberg, D. H., Mortonson, M. J., Eisenstein, D. J., et al. 2013, PhR, 530, 87

Yoon, S.-C., Podsiadlowski, P., \& Rosswog, S. 2007, MNRAS, 380, 933

Zhu, C., Chang, P., van Kerkwijk, M. H., \& Wadsley, J. 2013, ApJ, 767, 164

Zhu, C., Pakmor, R., van Kerkwijk, M. H., \& Chang, P. 2015, ApJL, 806, L1 